\title{
Fundamental Limits on Detection of UAVs by Existing Terrestrial RF Networks
}

This paper was downloaded from TechRxiv (https://www.techrxiv.org).

\section{LICENSE}

$\mathrm{CCO}$

SUBMISSION DATE / POSTED DATE

28-08-2021 / 01-09-2021

\section{CITATION}

Sinha, Priyanka; Guvenc, Ismail; Gursoy, Mustafa Cenk (2021): Fundamental Limits on Detection of UAVs by Existing Terrestrial RF Networks. TechRxiv. Preprint. https://doi.org/10.36227/techrxiv.16529592.v1

$\mathrm{DOI}$

10.36227/techrxiv.16529592.v1 


\title{
Fundamental Limits on Detection of UAVs by Existing Terrestrial RF Networks
}

\author{
Priyanka Sinha, İsmail Güvenç, and M. Cenk Gürsoy
}

\begin{abstract}
Detection of drones carries critical importance for safely and effectively managing unmanned aerial system traffic in the future. Given the ubiquitous presence of the drones across all kinds of environments in the near future, wide area drone detection and surveillance capability are highly desirable, which require careful planning and design of drone sensing networks. In this paper, we seek to meet this need by using the existing terrestrial radio frequency (RF) networks for passive sensing of drones. To this end we develop an analytical framework that provides the fundamental limits on the network-wide drone detection probability. In particular, we characterize the joint impact of the salient features of the terrestrial RF networks, such as the spatial randomness of the node locations, the directional 3D antenna patterns, and the mixed line of sight/non line of sight (LoS/NLoS) propagation characteristics of the air-to-ground (A2G) channels. Since the strength of the drone signal and the aggregate interference in a sensing network are fundamentally limited by the 3D network geometry and the inherent spatial randomness, we use tools from stochastic geometry to derive the closed-form expressions for the probabilities of detection, false alarm and coverage. This, in turn, demonstrates the impact of the sensor density, beam tilt angle, half power beam width (HPBW) and different degrees of $\mathrm{LoS}$ dominance, on the projected detection performance. Our analysis reveals optimal beam tilt angles, and sensor density that maximize the network-wide detection of the drones.
\end{abstract}

Index Terms-A2G channel, beam tilt, directional antenna pattern,

drone detection, LoS/NLoS, stochastic geometry.

\section{INTRODUCTION}

Due to the widespread use of drones across the military, commercial, and government sectors, surveillance of drones is emerging as an important and challenging problem. While such proliferation of drones introduces obvious benefits, it also increases the potential for accidental or deliberate violations, such as, invading privacy of people, intruding into restricted government or business areas, or colliding with air-crafts and causing accidents. In situations like these, technologies to detect, track, and interdict possible aerial threats become indispensable [1]. Since the detection of the drones is the first enabling step for the rest of the surveillance measures to take place, robust detection of the drones is of fundamental importance. Moreover, due to the pervasive presence of the drones in today's world, the wide area coverage of drone detection capabilities is also highly desirable.

P. Sinha, and İ. Güvenç are with the Dept. Electrical and Computer Engineering, NC State University, Raleigh, NC, 27606 (e-mail: \{psinha2, iguvenc\}@ncsu.edu). M. C. Gürsoy is with the Dept. Electrical Engineering and Computer Science, Syracuse University, Syracuse, NY, 13210 (email: megursoy@syr.edu).

This work has been supported by NASA under the Award NX17AJ94A.
Most state of the art drone detection methods typically use one of the following modalities or a fusion of them: ambient radio frequency (RF) signals, radar, acoustic signals, and computer vision. Among these, a good majority of the sensing techniques rely on the use of the RF signals, due to some of the advantages it offers over the other modalities, such as, the long detection range, effectiveness in NLoS environments, and the low-cost of the RF sensors [2]. The existing RF drone sensing techniques can be broadly classified into the following two categories: active sensing and passive sensing. In the active approach, the sensors are required to continuously broadcast wireless signals, and then analyze the signals reflected from the drone, to discover any unique signatures associated with a drone, such as, the ones caused by the drone's propellers, or the vibration patterns of the drone's body. In the passive approach, the sensor eavesdrops on the communication signal exchanged between the drones and their ground controllers (GCs) and looks for signatures unique to drones, such as the spectral features, traffic pattern, and the communication rate between the drones and their GCs.

While extensive research [1]-[4] has been carried out that focus on the signature analysis techniques, these studies make the fundamental assumption that the target drone is within the range of the said sensors and that the RF interference at the sensor's receiver is low enough for the sensor to reliably detect the presence of an aerial object. Since the detection performance is fundamentally limited by the intensity of the drone's signal at the sensor and the level of possible RF interference [1] at the sensor, careful system design is required to ensure sufficient signal-to-interference (SIR) level, before the signature analysis techniques can be put into practice. Especially in dense urban areas, with large number of mobile users, such high SIR levels can be extremely difficult to achieve. The detection performance can further suffer in such areas, as the probability of having a LoS link towards the drone is generally low due to high buildings. One of the ways to achieve a high SIR and a high probability of LoS, is by employing a dense and pervasive network of sensors. While the shorter A2G radio links in a dense sensor network improve the probability of detection and false alarm, the dense deployment also improves the area coverage probability [5].

Although dedicated RF sensor networks for drone detection can be deployed in a limited stretch within a region of interest (ROI), it cannot achieve the desired wide-area coverage. To this end, we propose the use of the existing terrestrial RF infrastructures that operate in the same frequency as the target drone. Drones are expected to widely use the common cellular network frequencies in the future for command, control, 


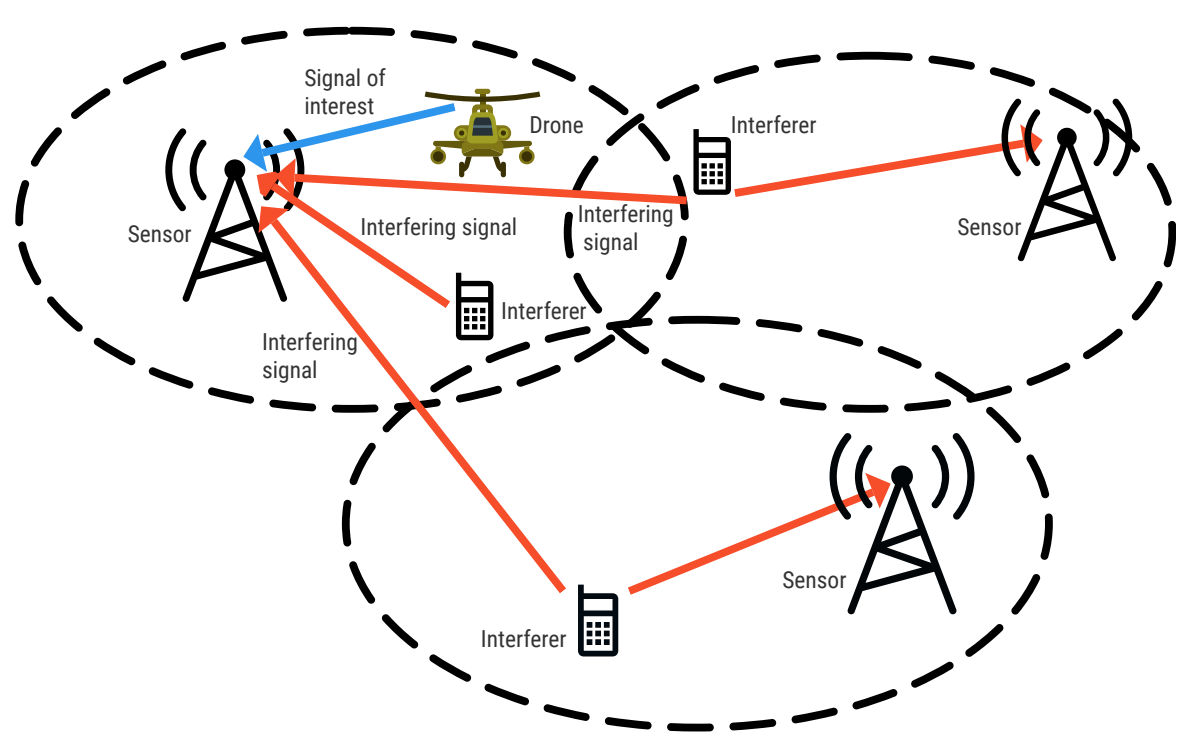

Fig. 1: The signal of interest from the drone and the interference at the probe sensor.

and payload communications [6]-[10]. Moreover, given the anticipated high density of the long term evolution unlicensed (LTE-U) base stations (BSs) in the $5 \mathrm{G}$ era, it is possible to use these resources for passive sensing of the commercial off the shelf (COTS) drones, that use WiFi links to communicate with their GCs. Since COTS drones are widely used across different applications, and both WiFi devices and LTE-U BSs operate in the unlicensed industrial, scientific and medical (ISM) radio bands [11], such detection framework remains applicable to a large group of drones. In the rest of the paper we do not limit the terrestrial sensing network to a specific technology, and provide a generic framework that is applicable to drones that may operate in either the cellular and ISM bands.

Since the legacy terrestrial networks are not primarily designed for drone detection, we need to account for the features that are unique to these networks and impact the signal intensity as well as the interference level. In particular, the drone's signal strength and the interference from the intended users of the sensors depend largely on the spatial locations of the sensors relative to the target drone and the users. Other factors that may impact the fading of the signals significantly, are the directionality of the antenna patterns (for example, cellular BSs usually have a tilted antenna pattern) and the degrees of LoS dominance in the A2G and the terrestrial channels. Thus the coupling of the 3D antenna patterns, and the propagation characteristics with the topological randomness of the network need to be considered explicitly using tools from stochastic geometry.

Notation: $X \sim \mathcal{U}[a, b]$ denotes that the scalar random variable $X$ is uniformly distributed between $a$ and $b . X \sim \chi_{2}^{2}$ and $X \sim \sigma \chi_{2}^{2}$ denote a standard (unit variance) and a scaled (variance of $\sigma^{2}$ ) Chi-squared random variable with two degrees of freedom. $X \sim \chi_{2}^{\prime 2}\left(\lambda_{\mathrm{nc}}\right)$ and $X \sim \sigma \chi_{2}^{\prime 2}\left(\lambda_{\mathrm{nc}}\right)$ denote a standard (unit variance and non-centrality parameter $\lambda_{\mathrm{nc}}$ ) and a scaled (variance of $\sigma^{2}$ and non-centrality parameter $\sigma \lambda_{\text {nc }}$ ) NonCentral Chi-squared random variable of degree 2. $\boldsymbol{X}$ is used to denote a complex random variable. $X \stackrel{d}{\rightarrow} Y$ denotes that the random variable converges to the random variable $Y$ in distribution. $a(\bmod b)$ is used to describe the modulo operation and the result is the remainder of the Euclidean division $\frac{a}{b}$.

\section{LITERATURE REVIEW}

Recently some works have emerged that investigate the topic of drone detection by cellular networks. Papers such as [12], [13] discuss application of various machine learning algorithms such as logistic regression, decision tree and $\mathrm{KNN}$, where the data is composed of radio measurements collected from the cellular networks. However, the authors of these papers only report the detection performance yielded by the said machine learning algorithms, and do not build a theoretical framework that can characterize the impact of the various network and RF parameters, such as the node density, the the drone altitude, and the beam width and the boresight angles of the antenna patterns at the BS receivers, on the detection performance. In [14], [15] the authors describe various enabling technologies and algorithms that allow the $5 \mathrm{G}$ infrastructure to be leveraged for drone detection. Both of these papers reply on the use of $5 \mathrm{G}$ millimeter wave (mmWave) BSs, as radars, and thus are not applicable to studying the detection performance of a passive sensing scheme. Further, they also lack a theoretical framework for the analysis of the projected detection performance. In our work we used tools from stochastic geometry to build such an analytical framework dedicated to detection of drones by existing cellular networks.

Most of the related stochastic geometry-based literature aims to study the coverage probability in the following two main scenarios, that involve drones: 1) drone-assisted aerial cellular networks serving ground users; and 2) ground cellular network serving drone users. For example, papers such as [16]-[18], evaluate the cellular coverage performance of drone assisted aerial cellular networks, without considering any realistic $\mathrm{A} 2 \mathrm{G}$ channel characteristics and antenna patterns. While the authors in [19], study the impact of a mixed LoS/NLoS A2G channel on the performance of a droneassisted heterogeneous networks, possible impacts of 3D antenna patterns were ignored. Some other recent papers [20], 
[21] considered dedicated ground BSs serving a network of drones under practical 3D antenna patterns. While the cellular coverage performance of the network was studied in these recent works, passive sensing performance of drones has not been taken into account. Since the characterization of the signal and the interference is completely different in a passive sensing scenario, none of these existing papers can be directly applied to a non-dedicated passive sensing network that we consider in our study.

In our work, we develop a unified analytical framework which jointly takes into account the impact of the random network geometry, the mixed LoS/NLoS A2G propagation characteristics, and the 3D directional antenna patterns on the detection performance of a non-dedicated RF sensing network. To the best of our knowledge, such an analytical framework motivating the use of the existing RF infrastructures is not available in the literature. This study is an extended version of our conference paper [22], where we investigated the performance of a sensor network with an isotropic antenna pattern. In the journal version, we incorporate a realistic directional antenna pattern, defined by the International Telecommunication Union (ITU) for a cellular network [23], that takes both the beam tilt angle and the HPBW into account. We also consider an $\mathrm{A} 2 \mathrm{G}$ channel model that allows us to differentiate between the detection performance in suburban and urban environments. Our analysis reveals several useful network design insights, such as, the existence of a critical sensor density and a critical beam tilt angle that optimize the detection coverage. We also demonstrate how the coverage probability of detection is impacted by the choice of the probe sensor (i.e. whether to consider detection by the nearest sensor or the $n^{\text {th }}$ nearest sensor), and how the most favorable choice is dependent on the sensor density, the drone altitude, the beam tilt, the HPBW, and the A2G propagation characteristics.

For easier comparison, we present a brief summary of the literature review section in Table.I. This table includes the following abbreviations that have not been used earlier in this manuscript: 'BPPP' for binomial Poisson point process, 'SDR' for software defined radio, 'ULA' for uniform linear array, ' $\mathrm{Y}$ ' for yes, and 'N/A' for not applicable.

\section{System MODEL}

\section{A. Network Overview}

As shown in Fig. 1, we consider a scenario in which a target drone is flying over a network of ground RF sensors. In the rest of the paper, we use the term sensor in a generic sense, to refer to a transceiver that is capable of sensing the band where the drones also communicate. While the ground sensors are serving their own intended user equipments (UEs), they aim to detect the target drone in the presence of the uplink transmissions from the UEs. Our assumption is that the drone transmission is co-channel with the ground sensors (such as LTE-U BSs) and the respective UE transmissions, as the drone uses a co-channel RF link (such as a WiFi link in the case of a COTS drone) for command, control, and communications with its GC. From the detection point of view, the active ground UEs therefore act as a network of interferers, and their uplink

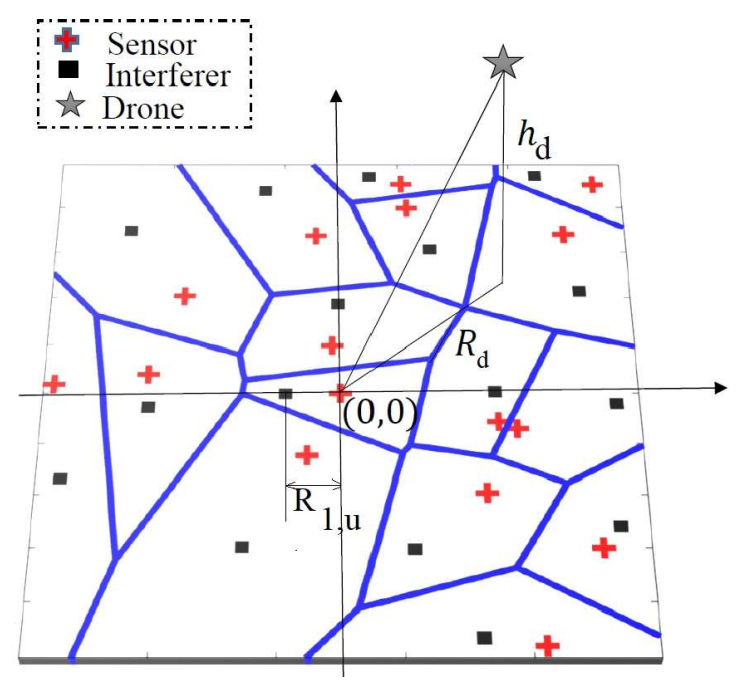

Fig. 2: The problem of drone detection in a Poisson field of sensors and interferers.

signals constitute the network interference, while the signal of interest (SOI) to be detected is captured from the target drone.

We assume that the sensors and UEs are deployed on the horizontal plane at heights of $h_{\mathrm{s}}$ and $h_{\mathrm{ue}}$ following two independent stationary homogeneous Poisson point processes (HPPPs) [24], denoted by $\Phi_{\mathrm{B}}$ and $\Phi_{\mathrm{u}}$, with the densities $\lambda \mathrm{m}^{-2}$ and $k_{\mathrm{u}} \lambda \mathrm{m}^{-2}$, respectively, where $k_{\mathrm{u}}>0$ represents the user load of each sensor. Note that any sensor can serve multiple users in single time-frequency resource block (RB) depending on the underlying multiuser-detection (MUD) capability, in which case user load becomes an integer such that $k_{\mathrm{u}} \geq 2$, and may be partially loaded which makes $k_{\mathrm{u}}$ a positive fraction $\left(k_{\mathrm{u}} \leq 1\right)$. We assume that the probe sensor, which is tasked with detecting the target drone, is located at the origin, as depicted in Fig. 2. The distance of the $i$-th nearest UE to the probe sensor is denoted by $R_{i, \mathrm{u}}$ such that $R_{k, \mathrm{u}} \leq R_{\ell, \mathrm{u}}$ for $k<\ell$. We further assume that each UE is associated with its nearest sensor, and hence the probability density function (PDF) of the distance of the intended UE to the probe sensor (i.e., $R_{1, \mathrm{u}}$ ) is [25]:

$$
f_{R_{1, \mathrm{u}}}\left(r_{1, \mathrm{u}}\right)=2 \pi k_{\mathrm{u}} \lambda r_{1, \mathrm{u}} \exp \left(-k_{\mathrm{u}} \lambda \pi r^{2}{ }_{1, \mathrm{u}}\right),
$$

which describes the Rayleigh distribution with a scale parameter $\sigma^{2}=\frac{1}{\pi k_{\mathrm{u}} \lambda}$.

Considering the tilted directional antenna pattern at the sensors, we take into account the detection of the target drone not only at the nearest sensor but also by the farther ones. Note that the natural choice would be to consider the detection at the nearest sensor if the antenna power pattern was perfectly isotropic [22]. As the antenna power pattern becomes nonisotropic, some farther sensors can receive a better-quality SOI. Thus for the purpose of generality, we consider detection of the target drone by its $n$-th nearest sensor, and the PDF of the horizontal distance between the probe sensor and the ground projection of the 3D location of the drone (denoted as the random variable $R_{\mathrm{d}}$ ) is given as [25], pp. 41:

$$
f_{R_{\mathrm{d}}}\left(r_{\mathrm{d}}\right)=\frac{2 \pi^{n}}{\Gamma(n)} \lambda^{n} r_{\mathrm{d}}^{2 n-1} \exp \left(-\lambda \pi r_{\mathrm{d}}^{2}\right),
$$


TABLE I: Related work on RF-based detection/communication involving drones.

\begin{tabular}{|c|c|c|c|c|c|c|c|c|}
\hline Paper & Task & Infrastructure & $\begin{array}{l}\text { Operating } \\
\text { frequency }\end{array}$ & $\begin{array}{l}\text { Type of anal- } \\
\text { ysis }\end{array}$ & $\begin{array}{l}\text { Interference } \\
\text { geometry }\end{array}$ & $\begin{array}{l}\text { Sensor } \\
\text { geometry }\end{array}$ & $\begin{array}{l}\text { Channel } \\
\text { model }\end{array}$ & $\begin{array}{l}\text { Antenna pat- } \\
\text { tern }\end{array}$ \\
\hline [1] & $\begin{array}{l}\text { Passive sensing } \\
\text { of drones }\end{array}$ & $\begin{array}{l}\text { Surveillance } \\
\text { drones }\end{array}$ & N/A & Simulation & N/A & N/A & LoS/NLoS & N/A \\
\hline$[2,4]$ & $\begin{array}{l}\text { Detection } \\
\text { classification of } \\
\text { drones }\end{array}$ & $\begin{array}{l}\text { COTS ground } \\
\text { sensor }\end{array}$ & $\begin{array}{lr}2.4 & \mathrm{GHz} \\
\mathrm{N} / \mathrm{A}, 2.4 \mathrm{GHz}\end{array}$ & Experiments & N/A & N/A & N/A & N/A \\
\hline [11] & $\begin{array}{l}\text { Active / passive } \\
\text { sensing of drones }\end{array}$ & $\begin{array}{ll}\text { COTS } & \text { SDR } \\
\text { boards } & \end{array}$ & $2.437 \mathrm{GHz}$ & Experiments & N/A & N/A & N/A & N/A \\
\hline [12] & $\begin{array}{l}\text { Detection } \\
\text { classification of } \\
\text { drones }\end{array}$ & $\begin{array}{l}\text { Cellular net- } \\
\text { work }\end{array}$ & $2 \mathrm{GHz}$ & Simulation & N/A & N/A & LoS & $\begin{array}{l}\text { Extended 3GPP } \\
\text { Downtilted an- } \\
\text { tenna pattern }\end{array}$ \\
\hline [13] & $\begin{array}{lr}\text { Detection } \\
\text { identification of } \\
\text { drones }\end{array}$ & $\begin{array}{l}\text { Cellular net- } \\
\text { work }\end{array}$ & $2 \mathrm{GHz}$ & Simulation & N/A & $\begin{array}{l}\text { 3GPP-based de- } \\
\text { ployment }\end{array}$ & LoS/NLoS & N/A \\
\hline [14] & $\begin{array}{lr}\text { Detection } \\
\text { identification of } \\
\text { drones }\end{array}$ & $\begin{array}{l}5 \mathrm{G} \text { millimeter } \\
\text { wave radar }\end{array}$ & $25 \mathrm{GHz}$ & Simulation & N/A & $\begin{array}{l}5 \mathrm{G} \text { network de- } \\
\text { ployment }\end{array}$ & mmWave LoS & N/A \\
\hline [15] & $\begin{array}{l}\text { Detection } \\
\text { identification of } \\
\text { drones }\end{array}$ & $\begin{array}{l}\text { 5G millimeter } \\
\text { wave radar }\end{array}$ & $28 \mathrm{GHz}$ & $\begin{array}{l}\text { Ray based } \\
\text { tools }\end{array}$ & $\begin{array}{l}\text { Fixed } \\
\text { numbered, } \\
\text { randomly } \\
\text { located }\end{array}$ & Single BS & mmWave LoS & $\begin{array}{l}\text { Antenna array } \\
\text { beams }\end{array}$ \\
\hline$[16,17]$ & $\begin{array}{l}\text { Downlink cellu- } \\
\text { lar coverage at } \\
\text { ground receiver }\end{array}$ & $\begin{array}{l}\text { Aerial cellu- } \\
\text { lar network }\end{array}$ & N/A & $\begin{array}{l}\text { Stochastic Ge- } \\
\text { ometry }\end{array}$ & BPPP & N/A & $\begin{array}{l}\text { Nakagami-m } \\
\text { fading }\end{array}$ & N/A \\
\hline [18] & $\begin{array}{l}\text { Downlink cellu- } \\
\text { lar coverage at }\end{array}$ & $\begin{array}{l}\text { Aerial cellu- } \\
\text { lar network }\end{array}$ & N/A & $\begin{array}{l}\text { Stochastic } \\
\text { geometry } \\
\text { based mobility } \\
\text { model }\end{array}$ & PPP & N/A & Rayleigh fading & N/A \\
\hline [19] & $\begin{array}{l}\text { Downlink cellu- } \\
\text { lar coverage at }\end{array}$ & $\begin{array}{l}\text { Drone small } \\
\text { cells }\end{array}$ & sub-6 GHz & $\begin{array}{l}\text { Stochastic ge- } \\
\text { ometry }\end{array}$ & PPP & N/A & LoS/NLoS & N/A \\
\hline$[20,21]$ & $\begin{array}{l}\text { Downlink cellu- } \\
\text { lar coverage at }\end{array}$ & $\begin{array}{l}\text { Ground BS } \\
\text { and UAV-UEs }\end{array}$ & N/A & $\begin{array}{l}\text { Stochastic ge- } \\
\text { ometry }\end{array}$ & PPP & N/A & LoS/NLoS & $\begin{array}{l}\text { Downtilted } \\
\text { ULA pattern }\end{array}$ \\
\hline $\begin{array}{l}\text { Our } \\
\text { work }\end{array}$ & $\begin{array}{l}\text { Passive sensing } \\
\text { of drones }\end{array}$ & $\begin{array}{l}\text { Cellular net- } \\
\text { work }\end{array}$ & $3.5 \mathrm{GHz}$ & $\begin{array}{l}\text { Stochastic ge- } \\
\text { ometry }\end{array}$ & PPP & PPP & LoS/NLoS & $\begin{array}{l}\text { Downtilted an- } \\
\text { tenna beam }\end{array}$ \\
\hline
\end{tabular}

which is the $n$-th nearest neighbor distance of the HPPP, $\Phi_{\mathrm{B}}$. Note that (2) describes the generalized Gamma distribution with the scale parameter $\sigma^{2}=\frac{1}{\pi \lambda}$.

\section{B. A2G Propagation Channels}

Due to the higher altitude of the drones, the $A 2 \mathrm{G}$ channels are more likely to have a LoS component as compared to the terrestrial links. The probability of the A2G link having a LoS component (i.e., LoS state), is defined as a function of the horizontal distance $r_{\mathrm{d}}$ as follows [26]:

$$
\mathrm{P}_{\text {LoS }}^{\mathrm{A}}\left(r_{\mathrm{d}}\right)=\frac{1}{1+a \exp \left\{-b\left[\frac{180}{\pi} \tan ^{-1}\left(\frac{h_{\mathrm{d}}-h_{\mathrm{s}}}{r_{\mathrm{d}}}\right)-a\right]\right\}},
$$

where $a$ and $b$ are constants which depend on the environment, $h_{\mathrm{d}}$ is the altitude of the target drone, and $\beta_{\mathrm{d}}=\tan ^{-1}\left(\left[h_{\mathrm{d}}-h_{\mathrm{s}}\right] / r_{\mathrm{d}}\right)$ is the UAV elevation angle, or equivalently the elevation-angle-of-arrival (AOA) of the respective LoS link at the sensor. Note that the channel is composed of only the NLoS links (i.e., NLoS state) with the probability $1-\mathrm{P}_{\text {LoS }}^{\mathrm{A}}\left(r_{\mathrm{d}}\right)$. As illustrated by Fig. 3, the NLoS component is assumed to be composed of $M_{\mathrm{d}}$ multipath components (MPCs), where each path is characterized by a random amplitude gain $\alpha_{m}$ with unit mean-squared value $\left(\mathbb{E}\left\{\alpha_{m}^{2}\right\}=1\right)$ [27], a random phase $\phi_{m}\left(\phi_{m} \sim \mathcal{U}[0,2 \pi)\right)$, and a random AOA $\beta_{m}$ at the probe sensor $\left(\beta_{m} \sim \mathcal{U}\left[-\frac{\pi}{2}, \frac{\pi}{2}\right]\right)$. We also assume that $\left\{\alpha_{m}\right\},\left\{\beta_{m}\right\}$, and $\left\{\phi_{m}\right\}$ are independent and identically distributed (iid) random variables with $1 \leq m \leq M_{\mathrm{d}}$
[28], [29]. Note that the A2G links can have MPC contributions scattered by obstructions located both above and below the ground sensor, and we therefore have: $-\frac{\pi}{2} \leq \beta_{m} \leq \frac{\pi}{2}$.

Depending on the A2G channel state $\mathrm{s}_{\mathrm{A}}$ being LoS or NLoS (i.e., $\mathrm{s}_{\mathrm{A}} \in\{\operatorname{LoS}, \mathrm{NLoS}\}$ ), the large-scale fading in the said A2G channel is characterized as the combination of free space path loss (FSPL) and respective additional path loss $(\triangle P L) \eta^{\mathrm{s}_{\mathrm{A}}}$, arising from the shadowing and scattering caused by the environmental obstacles (e.g., buildings, lampposts). We assume different additional path loss for the LoS and the NLoS links such that $\eta^{\mathrm{NLOS}}>\eta^{\mathrm{LoS}}$, in general. Thus, the path loss over the A2G link is given in the $\mathrm{dB}$ scale as follows

$$
\mathrm{PL}^{\mathrm{S}_{\mathrm{A}}}=\underbrace{20 \log \left(d_{\mathrm{LoS}}\right)+20 \log \left(\frac{4 \pi f_{\mathrm{c}}}{\mathrm{c}}\right)}_{\text {FSPL }}+\underbrace{20 \log \left(\eta^{\mathrm{S}_{\mathrm{A}}}\right)}_{\text {LoS/NLOS } \Delta \mathrm{PL}},
$$

where $d_{\text {LoS }}=\sqrt{r_{\mathrm{d}}^{2}+\left(h_{\mathrm{d}}-h_{\mathrm{s}}\right)^{2}}$ is the LoS distance between the probe sensor and the target drone, and $\mathrm{c}$ is the speed of light. We use (4) for describing the LoS and NLoS components of received drone signal in (16) and (17).

\section{Terrestrial Propagation Channels}

In the terrestrial channels between the sensors and UEs, the probability of having a LoS component is generally lower as compared to the A2G links, especially over long propagation distances (e.g., between the probe sensor and 


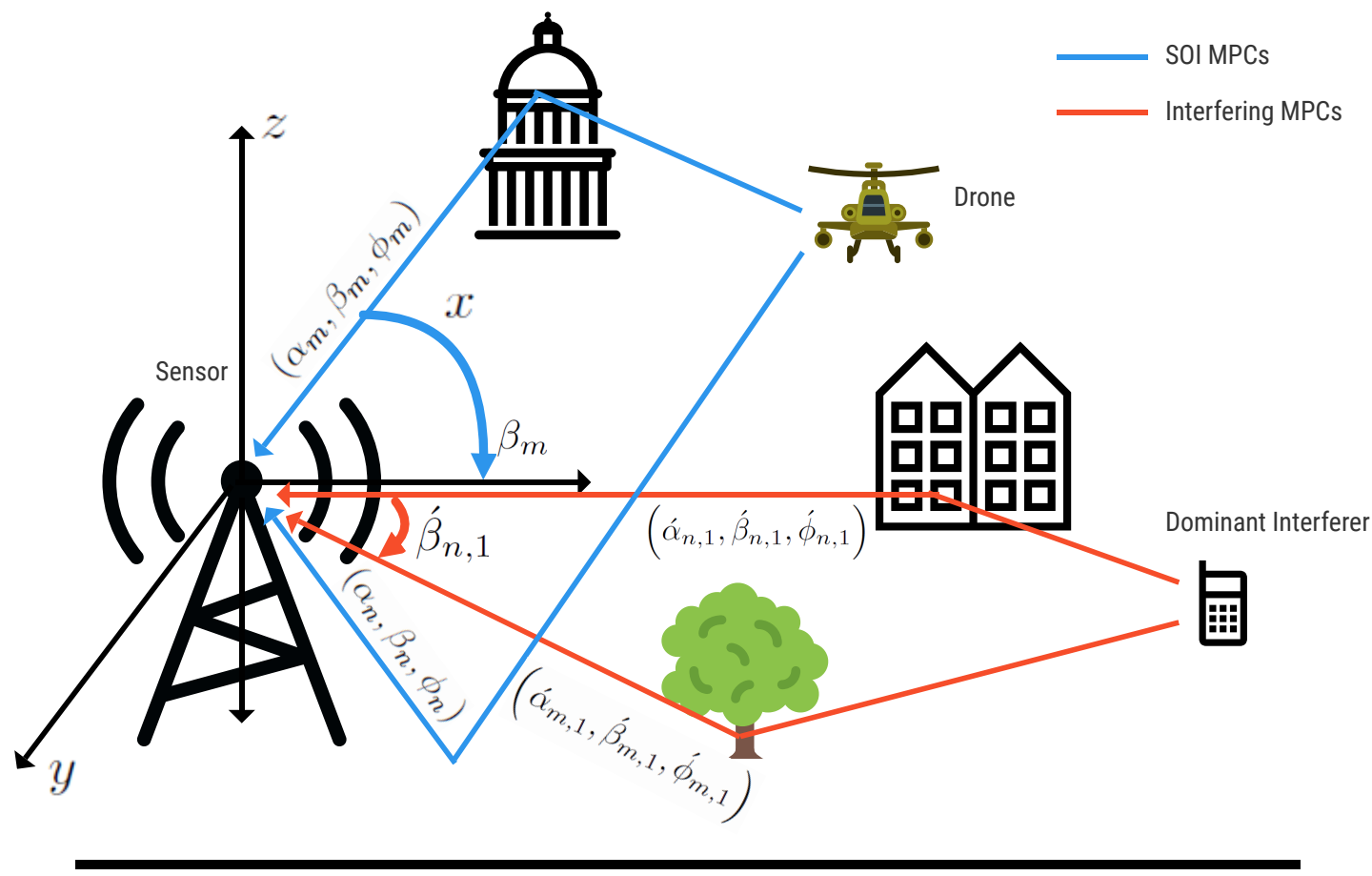

Fig. 3: NLoS propagation in $\mathrm{A} 2 \mathrm{G}$ and terrestrial channels.

distant interfering UEs-other than the nearest UE). On the other hand, the LoS probability becomes significant for shortdistance connections (e.g., between the probe sensor and its intended UE). We therefore describe the terrestrial channel between the probe sensor and intended UE as a mixture of LoS and NLoS links (similar to A2G channels), and assume NLoS-only links for the channels between the probe sensor and interfering UEs. Adopting the urban micro-cellular (UMi) street canyon model of the 3rd Generation Partnership Project (3GPP) [30], we assume that the intended UE link can either be in $\operatorname{LoS}$ state or NLoS state (st $\in\{L O S, N L o S\})$ and the probability of the link being in the LoS state is given as a function of the horizontal distance between the probe sensor and its intended UE as

$$
\begin{aligned}
& \mathrm{P}_{\text {LoS }}^{\top}\left(r_{1, \mathrm{u}}\right)=\min \left(\frac{18}{r_{1, \mathrm{u}}}, 1\right)\left(1-\exp \left\{-\frac{r_{1, \mathrm{u}}}{36}\right\}\right) \\
& +\exp \left\{-\frac{r_{1, \mathrm{u}}}{36}\right\} .
\end{aligned}
$$

We therefore assume that the intended UE has both the LoS and NLoS links (i.e., mixed LoS/NLoS channel) with a probability in (5), and has only NLoS links otherwise. We use (5) for describing the LoS and NLoS components of the interfering signals in (15). As illustrated by Fig. 3, we assume that each NLoS link between each UE and the probe sensor, is composed of $M_{\mathrm{u}}$ MPCs and that each of these MPCs are characterized by a random amplitude gain $\dot{\alpha}_{m}$ with unit mean-squared value $\left(\mathbb{E}\left\{\dot{\alpha}_{m}^{2}\right\}=1\right)$, a random phase $\dot{\phi}_{m, i}$ $\left(\hat{\phi}_{m, i} \sim \mathcal{U}[0,2 \pi)\right)$, and a random AOA $\hat{\beta}_{m, i}\left(\hat{\beta}_{m, i} \sim \mathcal{U}\left[-\frac{\pi}{2}, 0\right]\right)$. Here, $i$ and $m$ respectively denote the UE order/index and the MPC index, and for all values of $i,\left\{\dot{\alpha}_{m, i}\right\},\left\{\dot{\beta}_{m, i}\right\}$, and $\left\{\hat{\phi}_{m, i}\right\}$ are sequences of random variables that are i.i.d. in both $1 \leq m \leq M_{\mathrm{u}}$ and $1 \leq i<\infty$.
Note that significant portion of the MPCs of the terrestrial links arrive at the ground sensor from below the horizon due to the ground UEs having height such that $h_{\text {ue }}<h_{\mathrm{s}}$, thereby producing AOAs in the range $\left[-\frac{\pi}{2}, 0\right]$. Note also that the AOA of the LoS link for the intended UE is given as $\beta_{1, \mathrm{u}}=-\tan ^{-1}\left(\left[h_{\mathrm{s}}-h_{\mathrm{ue}}\right] / r_{1, \mathrm{u}}\right)$. We also assume that the path loss in terrestrial LoS and NLoS links are respectively expressed by $r_{1, \mathrm{u}}^{\gamma_{\mathrm{L}}}$ and $r_{1, \mathrm{u}}^{\gamma_{\mathrm{L}}}$, where $\gamma_{\mathrm{L}}$ and $\gamma_{\mathrm{I}}$ are the respective path loss exponents (PLE).

\section{Impact of Directional Antenna Patterns}

A. 3D Antenna Pattern, Detection Zone, and Interference Zone

We assume that each UE and the target drone have been equipped with a single isotropic antenna. The ground sensors, on the other hand, have a 3D antenna pattern that are mechanically or electrically steered towards a certain elevation angle in the vertical domain. In this work, we adopt a directional antenna power pattern which is defined by the International Telecommunication Union (ITU) in the vertical domain as follows [23]

$$
G(\beta)=G_{\mathrm{P}, \max }-\min \left[12\left(\frac{\beta-\beta_{\mathrm{tilt}}}{\beta_{3 \mathrm{~dB}}}\right)^{2}, A_{\mathrm{m}}\right],
$$

where $\beta$ is the elevation angle (of either the ground UEs or target drone), $A_{\mathrm{m}}$ is the side-lobe level (SLL), $G_{\mathrm{P}, \max }$ is the maximum directional gain (along the boresight), $\beta_{\text {tilt }}$ is the beam tilt angle or boresight elevation angle, and $\beta_{3 \mathrm{~dB}}$ is the HPBW of the vertical antenna pattern at the probe sensor. We note that the antenna power pattern in (6) can be used for many different settings (e.g., 3GPP [30]) with a proper choice of model parameters.

Focusing on the signal reception through main lobe only, we realize that the elevation angles (either for the ground 


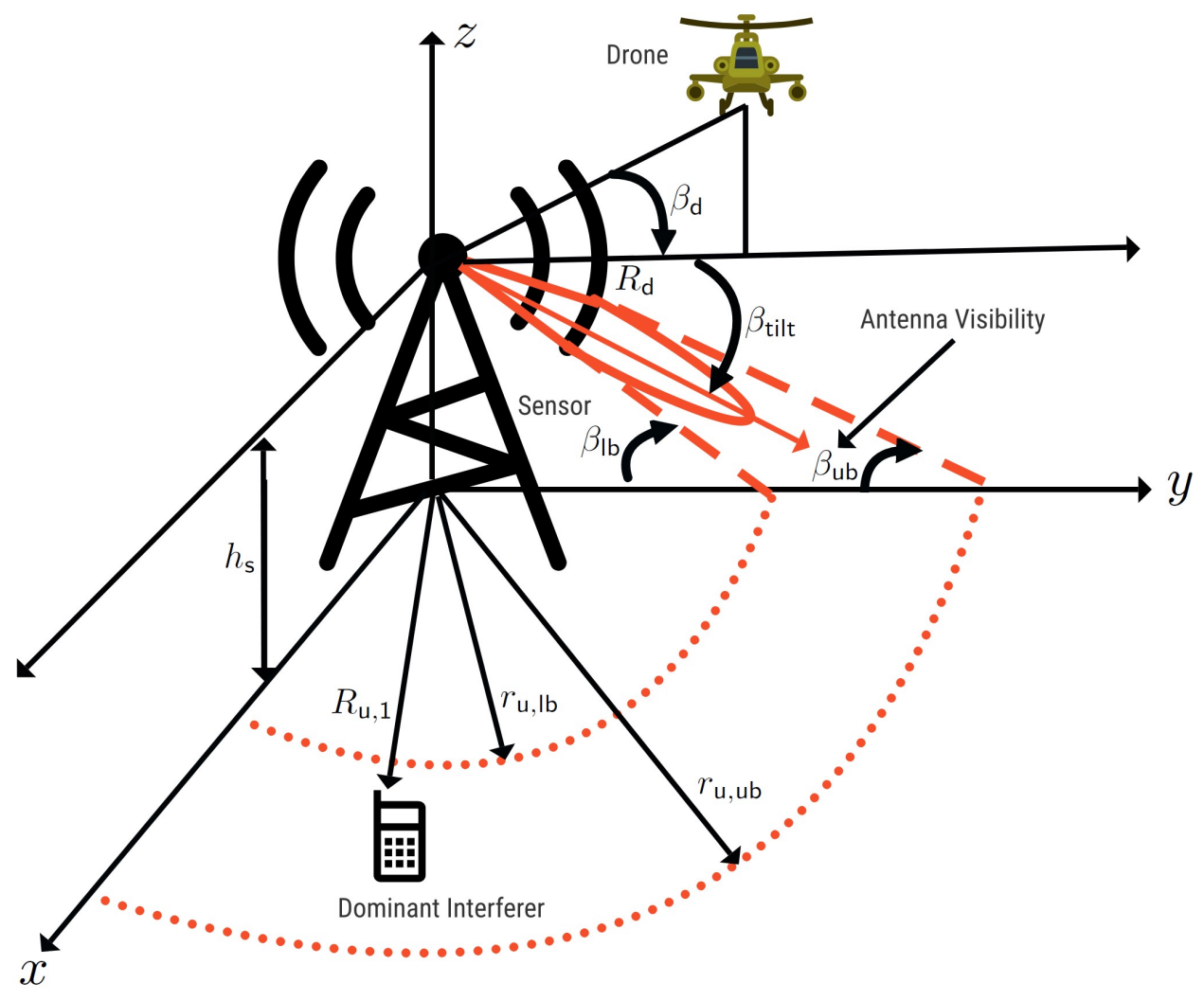

Fig. 4: Ground interference zone with respect to the dominant interferer for downtilt antenna.

UEs or target drone) in the range of $\beta_{\mathrm{lb}} \leq \beta \leq \beta_{\mathrm{ub}}$ yield a receive antenna gain higher than $\left(G_{\mathrm{P}, \max }-A_{\mathrm{m}}\right) \mathrm{dB}$, where $\beta_{\mathrm{lb}}=\beta_{\text {tilt }}-\sqrt{\frac{A_{\mathrm{m}}}{12}} \beta_{3 \mathrm{~dB}}$ and $\beta_{\mathrm{ub}}=\beta_{\mathrm{tilt}}+\sqrt{\frac{A_{\mathrm{m}}}{12}} \beta_{3 \mathrm{~dB}}$. Thus the intended ground UE can successfully (i.e. with a moderate to high receive gain) communicate with the probe sensor only if it is located at a suitable distance (i.e. all values of $r_{1, \mathrm{u}}$ for which $\beta_{\mathrm{lb}} \leq \beta_{1, \mathrm{u}} \leq \beta_{\mathrm{ub}}$ ) from the probe sensor. We refer to the collection of such nearest UE locations as the interference zone, as uplink signals received from other UE locations gets severely diminished due to experiencing the minimum receive gain of $-A_{\mathrm{m}} \mathrm{dB}$. Similarly, the SOI of the target drone can be successfully received by the probe sensor only when the ground projection of the drone's 3D location is inside a so-called detection zone, where we have $\beta_{\mathrm{lb}} \leq \beta_{\mathrm{d}} \leq \beta_{\mathrm{ub}}$. Apart from the beam tilt angle, the HPBW, and the height of the probe sensor, the area and location of the detection zone and the interference zone respectively depend on the altitude of the target drone and the intended ground UE. As shown in Fig. 4, we note that both of these zones form circular disks around the probe sensor, where the inner and outer radius of the interference zone are given respectively as $r_{\mathrm{u}, \mathrm{lb}}=\left(h_{\mathrm{s}}-h_{\mathrm{ue}}\right) \cot \left(\beta_{\mathrm{lb}}\right)$ and $r_{\mathrm{u}, \mathrm{ub}}=\left(h_{\mathrm{s}}-h_{\mathrm{ue}}\right) \cot \left(\beta_{\mathrm{ub}}\right)$, and those for the detection zone are $r_{\mathrm{d}, \mathrm{lb}}=\left(h_{\mathrm{d}}-h_{\mathrm{s}}\right) \cot \left(\beta_{\mathrm{ub}}\right)$ and $r_{\mathrm{d} \text {,ub }}=\left(h_{\mathrm{d}}-h_{\mathrm{s}}\right) \cot \left(\beta_{\mathrm{lb}}\right)$, respectively. Please note that, the reason we define the detection and the interference zone with respect to the antenna pattern, is that the detection performance is determined by the composite signal strength only, as opposed to the level of the signal-to-interference-ratio (SINR) in a communication scenario. In detection we only care about sensing the presence of the drone, and sensors are not required to decode the detected signal, as in the case of communication.

We define events $A$ and $B$ to describe the conditions in which the intended UE and target drone are in the interference zone and the detection zone, respectively. The probability of these events, i.e., $\mathbb{P}(A)=\mathbb{P}\left(\beta_{\mathrm{lb}} \leq \beta_{1, \mathrm{u}} \leq \beta_{\mathrm{ub}}\right)$ and $\mathbb{P}(B)=\mathbb{P}\left(\beta_{\mathrm{lb}} \leq \beta_{\mathrm{d}} \leq \beta_{\mathrm{ub}}\right)$, are the measures of how well the mainlobe is aligned towards the intended UE and the target drone, respectively. Using the respective horizontal distance distributions in (1) and (2), they are given as:

$$
\begin{gathered}
\mathbb{P}(A)= \begin{cases}\varphi_{\mathrm{A}}\left(\beta_{\mathrm{tilt}}-\beta_{3 \mathrm{~dB}}\right) \\
-\varphi_{\mathrm{A}}\left(\beta_{\mathrm{tilt}}+\beta_{3 \mathrm{~dB}}\right), & \beta_{\mathrm{lb}} \leq 0, \beta_{\mathrm{ub}} \leq 0 \\
\varphi_{\mathrm{A}}\left(\beta_{\mathrm{tilt}}+\beta_{3 \mathrm{~dB}}\right), & \beta_{\mathrm{lb}} \leq 0, \beta_{\mathrm{ub}} \geq 0 \\
0, & \beta_{\mathrm{lb}} \geq 0, \beta_{\mathrm{ub}} \geq 0\end{cases} \\
\mathbb{P}(B ; n)= \begin{cases}0, & \beta_{\mathrm{lb}} \leq 0, \beta_{\mathrm{ub}} \leq 0 \\
\varphi_{\mathrm{B}}\left(\beta_{\mathrm{tilt}}+\beta_{3 \mathrm{~dB}}\right), & \beta_{\mathrm{lb}} \leq 0, \beta_{\mathrm{ub}} \geq 0 \\
\varphi_{\mathrm{B}}\left(\beta_{\mathrm{tilt}}+\beta_{3 \mathrm{~dB}}\right) & \\
-\varphi_{\mathrm{B}}\left(\beta_{\mathrm{tilt}}-\beta_{3 \mathrm{~dB}}\right), & \beta_{\mathrm{lb}} \geq 0, \beta_{\mathrm{ub}} \geq 0\end{cases}
\end{gathered}
$$

where

$$
\begin{aligned}
\varphi_{\mathrm{A}}(x) & =\exp \left\{-\lambda \pi\left[\left(h_{\mathrm{s}}-h_{\mathrm{ue}}\right) \cot (x)\right]^{2}\right\}, \\
\varphi_{\mathrm{B}}(x) & =\sum_{s=0}^{n-1} \frac{\left[\lambda \pi\left(h_{\mathrm{d}}-h_{\mathrm{s}}\right)^{2} \cot ^{2}(x)\right]^{s}}{s !} \\
& \times \exp \left\{-\lambda \pi\left(h_{\mathrm{d}}-h_{\mathrm{s}}\right)^{2} \cot ^{2}(x)\right\} .
\end{aligned}
$$


Note that $\mathbb{P}(B ; n)$ denotes $\mathbb{P}(B)$ with the probe sensor being the $n$-th nearest sensor for the target drone, and $\varphi_{\mathrm{A}}(x)$ and $\varphi_{\mathrm{B}}(x)$ are defined as in (9).

\section{B. Numerical Example}

In order to get an insight into the probabilities in (7) and (8), we depict their values in Fig. 5a and Fig. 5b, respectively, against varying tilt angle $\beta_{\text {tilt }}$ for various choices of $3 \mathrm{~dB}$ beamwidth $\beta_{3 \mathrm{~dB}}$, node density $\lambda$, and drone altitude $h_{\mathrm{d}}$ assuming a particular antenna pattern with $A_{\mathrm{m}}=20 \mathrm{~dB}$, $G_{\mathrm{P}, \max }=17 \mathrm{dBi}, k_{\mathrm{u}}=1$, and $h_{\mathrm{ue}}=0$. In Fig. $5 \mathrm{~b}$, under the same configuration, $\mathbb{P}(B)$ is plotted for both the nearest ('N1') and the $2^{\text {nd }}$ nearest ('N2') sensor. Later in Section. VII-B, we demonstrate that, the impact of the varying $\beta_{\text {tilt }}$ on the detection performance is mainly governed by the corresponding changes in $\mathbb{P}(A)$ and $\mathbb{P}(B)$.

In view of both static and dynamic beamforming techniques, we assume that the mainlobe is steerable across the entire vertical plane. This allows us to explore the detection performance across a wider range of beam tilt angles including both negative and positive elevation angles, portraying respectively, a lower and an upper bound on the sensing performance, that is achievable by existing non-dedicated RF infrastructures. Moreover, with a rapid growth in the use of unmanned aerial systems (UASs) across various fields, the idea of installing RF infrastructures that are capable of serving both ground and aerial users are being studied heavily, which in turn makes such up-tilted directional antenna patterns more feasible. In Fig. 5a and Fig. 5b, as the tilt angle changes from $-30^{\circ}$ to $30^{\circ}$ and moves from the left to the right side of the $\beta_{\text {tilt }}$ axis, both $r_{\mathrm{u}, \mathrm{lb}}$ and $r_{\mathrm{u}, \mathrm{ub}}$ increase. This causes the interference zone to move away from the origin of the 2-D Euclidean plane, sweeping through the intervals of the intended UE distance, $R_{1, \mathrm{u}}$, that are low and high in probability density $\left(f_{R_{1, u}}\right)$. Thus in Fig. 5a we see that $\mathbb{P}(A)$ keeps increasing as long as the extent of the overlap between the interference zone and the high probability density interval of $R_{1, \mathrm{u}}$ (narrow neighborhood of the $\mathbb{E}\left\{R_{1, \mathrm{u}}\right\}$ ) increases, increasing the total probability mass (measured as $\left.\int_{r_{\mathrm{u}, \mathrm{ub}}}^{r_{\mathrm{u}}} f_{R_{1, \mathrm{u}}}\left(r_{1, \mathrm{u}}\right) \mathrm{d} r_{1, \mathrm{u}}\right)$ in the interference zone. However $\mathbb{P}(A)$ starts to drop, as $\beta_{\text {tilt }}$ increases beyond a critical angle that maximizes the total probability mass.

Similarly in Fig. $5 \mathrm{~b}$, we see that as the beam tilt angle increases beyond $\beta_{\mathrm{tilt}} \geq-\sqrt{\frac{A_{m}}{12}} \beta_{3 \mathrm{~dB}}$ (i.e. $\beta_{\mathrm{ub}} \geq 0$ ), the detection zone moves inward (i.e. both $r_{\mathrm{d}, \mathrm{lb}}$ and $r_{\mathrm{d} \text {,ub }}$ decrease), which in turn increases the overlap between the detection zone and the high probability density $\left(f_{R_{\mathrm{d}}}\left(r_{\mathrm{d}}\right)\right)$ intervals of the horizontal distance between the drone and the probe sensor, $R_{\mathrm{d}}$ (a narrow neighborhood of $\mathbb{E}\left\{R_{\mathrm{d}}\right\}$ ). This causes $\mathbb{P}(B)$ and the total probability mass $\left(\int_{r_{\mathrm{d}, \mathrm{bb}}}^{r_{\mathrm{d}}} f_{R_{\mathrm{d}}}\left(r_{\mathrm{d}}\right) \mathrm{d} r_{\mathrm{d}}\right)$ in the detection zone to increase until a certain critical tilt angle is reached and the detection zone moves too close to the origin reducing the total probability mass. Since the values of both $\mathbb{E}\left\{R_{1, \mathrm{u}}\right\}$ and $\mathbb{E}\left\{R_{\mathrm{d}}\right\}$ decrease with an increase in the sensor $(\lambda)$ and the UE $\left(k_{\mathrm{u}} \lambda\right)$ density, the high probability density intervals for both $R_{\mathrm{d}}$ and $R_{1, \mathrm{u}}$ move towards the origin. Thus in Fig. 5a, we see that, as $\lambda$ increases from $\lambda_{0}$ to $10 \lambda_{0}$ (where $\lambda_{0}=10^{-6} \mathrm{~m}^{-2}$ ), the tilt angle that maximizes $\mathbb{P}(A)$ moves towards the lower bound of the tilt angle range. Similarly, in Fig. 5b, as $\lambda$ increases, the tilt angle that maximizes $\mathbb{P}(B)$ moves towards the upper bound of the tilt angle range. Since a higher drone altitude also causes the elevation angle of the drone to become steeper, it also results in $\mathbb{P}(B)$ being maximized at a tilt angle that is closer to the upper bound of the tilt angle range. By observing Fig. 5a and by taking derivative of (7) with respect to $\beta_{\text {tilt }}$ and $\lambda$, the tilt angles for which $\frac{d \mathbb{P}(A)}{d \beta_{\text {titl }}}>0$, also yields $\frac{d \mathbb{P}(A)}{d \lambda}>0$, and the tilt angles for which $\frac{d \mathbb{P}(A)}{d \beta_{\text {titt }}}<0$, yields $\frac{d \mathbb{P}(A)}{d \lambda}<0$. Similarly, from Fig. $5 b$ and (8) we note that the tilt angles for which $\frac{d \mathbb{P}(B)}{d \beta_{\text {tilt }}}>0$, yields $\frac{d \mathbb{P}(B)}{d \lambda}<0$ and $\frac{d \mathbb{P}(B)}{d h_{\mathrm{d}}}<0$, whereas the tilt angles for which $\frac{d \mathbb{P}(B)}{d \beta_{\text {tilt }}}<0$, yields $\frac{d \mathbb{P}(B)}{d \lambda}>0$ and $\frac{d \mathbb{P}(B)}{d h_{\mathrm{d}}}>0$.

\section{Drone Detection in RF Sensing Network}

In this section, we formulate a binary hypothesis testing problem that detects the presence of a target drone by sensing any change in the distribution of the received signal strength (RSS) due to the presence of an A2G link between the probe sensor and the target drone. When the target drone is not present, which is referred to as the null hypothesis $\mathcal{H}_{0}$, the composite received signal $R(t)$ at the probe sensor is given by

$$
\mathcal{H}_{0}: R(t)=Y(t)+N(t),
$$

where $Y(t)$ is the aggregate interference and $N(t)$ is the additive white Gaussian noise (AWGN) with a two-sided power spectral density $N_{0} / 2$. In the presence of the target drone, the alternative hypothesis $\mathcal{H}_{1}$ is given by

$$
\mathcal{H}_{1}: R(t)=Z(t)+Y(t)+N(t),
$$

where $R(t)$ also involves the SOI $Z(t)$, in addition to $Y(t)$ and $N(t)$.

The aggregate interference term $Y(t)$ in (10) and (11) is given as [27]:

$$
Y(t)=Y_{1}(t)+\sum_{i=2}^{\infty} Y_{i, \mathrm{NLoS}}(t),
$$

where $Y_{1}(t)$ is the signal received at the probe sensor from the intended UE over the mixed LoS/NLoS link, and $Y_{i, \mathrm{NLoS}}(t)$ is the signal received from the $i$-th nearest UE (regardless of being the intended or interfering UE) over the NLoS links. The LoS component of $Y_{1}(t)$ is obtained by applying the receive antenna gain (according to the antenna pattern at the probe sensor) and large-scale fading (path loss) to the UE transmit signal as follows

$$
\begin{aligned}
Y_{1, \operatorname{LoS}}(t) & =\left(\frac{k}{R_{1, \mathrm{u}}}\right)^{\frac{\gamma_{\mathrm{L}}}{2}} \sqrt{2 P_{\mathrm{u}} G\left(\beta_{1, \mathrm{u}}\right)} g_{\mathrm{u}}\left(t-\tau_{1}\right) \\
& \times \cos \left(2 \pi f_{\mathrm{c}} t+\theta_{1, \mathrm{u}}\right) .
\end{aligned}
$$

Similarly, the NLoS component of the $i$-th nearest UE, for $i=$ $1,2, \ldots$, is computed after applying sensor antenna gain and both the small- and large-scale fading to UE transmit signal, which produces

$$
\begin{aligned}
Y_{i, \mathrm{NLoS}}(t) & =\sum_{m=1}^{M_{\mathrm{u}}} \alpha_{m, i}\left(\frac{k}{R_{i, \mathrm{u}}}\right)^{\frac{\gamma_{\mathrm{I}}}{2}} \sqrt{2 P_{\mathrm{u}} G\left(\dot{\beta}_{m, i}\right)} g_{\mathrm{u}}\left(t-\tau_{i}\right) \\
& \times \cos \left(2 \pi f_{\mathrm{c}} t+\theta_{\mathrm{i}, \mathrm{u}}+\dot{\phi}_{m, i}\right) .
\end{aligned}
$$




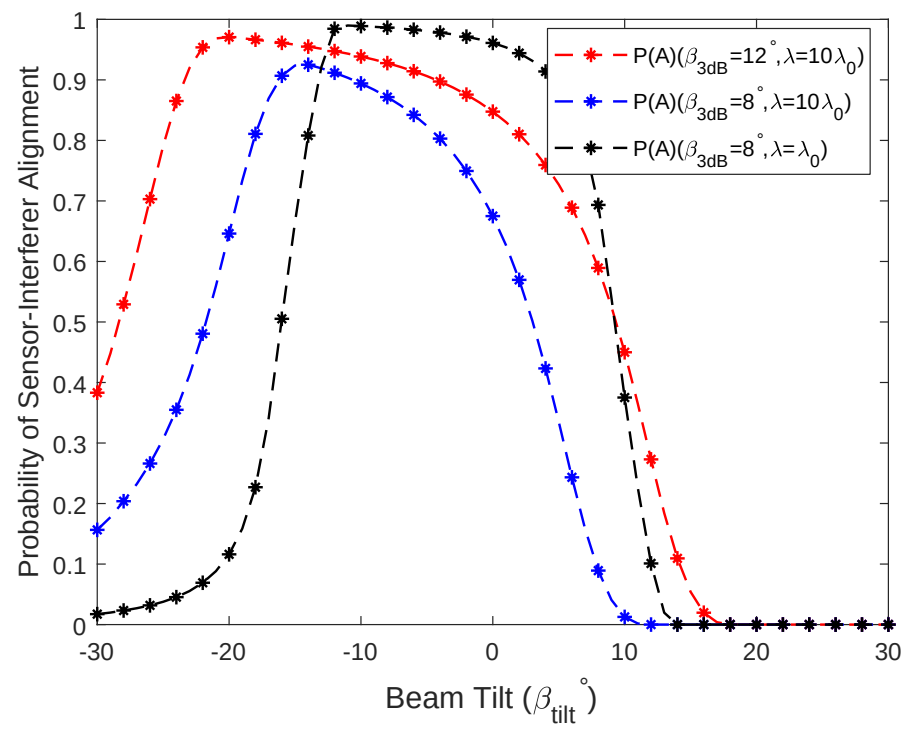

(a) Sensor-intended UE alignment.

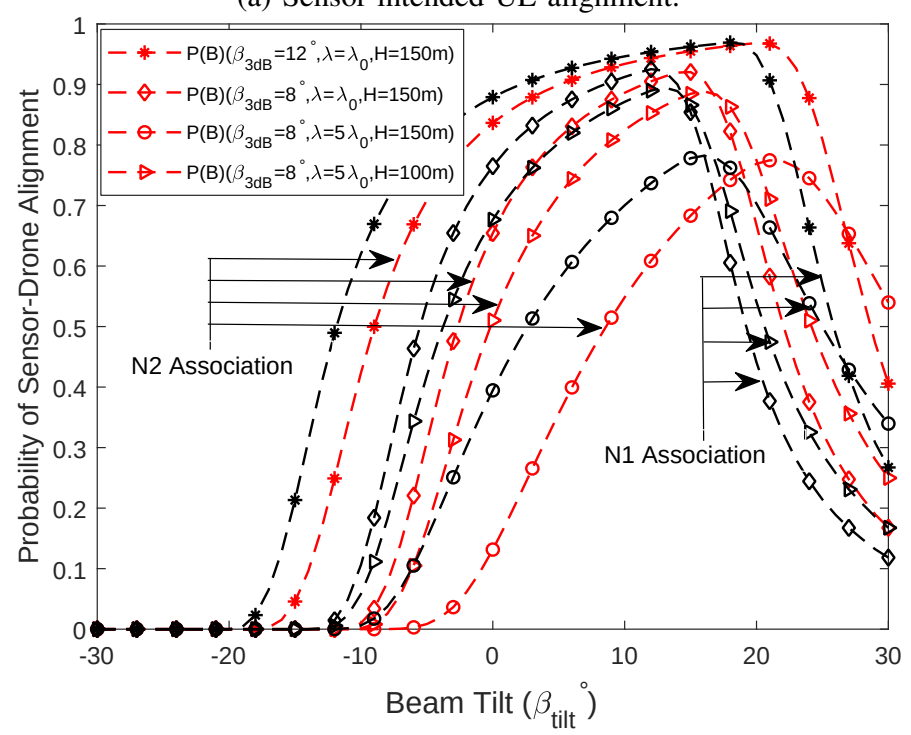

(b) Sensor-drone alignment.

Fig. 5: Probability of alignment with sensor antenna pattern as a function of the beam tilt.

Thus the total received signal for the intended UE is given by (13) and (14) as follows

$$
Y_{1}(t)=\left\{\begin{array}{ll}
Y_{1, \operatorname{LoS}}(t)+Y_{1, \mathrm{NLoS}}(t), & \text { with } \mathrm{P}_{\operatorname{LoS}}^{\top}\left(r_{1, \mathrm{u}}\right) \\
Y_{1, \mathrm{NLoS}}(t), & \text { with } 1-\mathrm{P}_{\mathrm{LoS}}^{\top}\left(r_{1, \mathrm{u}}\right)
\end{array},\right.
$$

where $\mathrm{P}_{\text {LoS }}^{\top}\left(r_{1, \mathrm{u}}\right)$ is the probability of the respective LoS state given in (5). In the above equations (13) and (14), we use the following notation: $f_{\mathrm{c}}$ is the carrier frequency, $\mathrm{c}$ is the speed of light, $k=\frac{\mathrm{c}}{4 \pi f_{\mathrm{c}}}, P_{\mathrm{u}}$ is the transmit power of the ground UEs, $g_{\mathrm{u}}(t)$ is the unit-energy signal waveform for the UEs, $\tau_{i}$ is the random delay in transmission of the $i$-th nearest $\mathrm{UE}$, and $\theta_{i, \mathrm{u}}$ is the signal phase of the $i$-th nearest UE taking values in the range $[0,2 \pi)$. In this analysis we do not require the ground UEs to be synchronized and allow them to transmit independently and asynchronously. In view of this, we assume $\left\{\tau_{i}\right\}$ and $\left\{\theta_{i, \mathrm{u}}\right\}$ to be two sequences of i.i.d random variables with $\tau_{i} \sim \mathcal{U}[0, T]$ and $\theta_{i, \mathrm{u}} \sim \mathcal{U}[0,2 \pi)$.

The SOI received at the probe sensor from the target drone is similarly described with its LoS and NLoS components. We obtain the LoS component by applying the sensor antenna pattern and large-scale fading to the drone's transmit signal as follows

$$
\begin{aligned}
Z_{\mathrm{LoS}}(t) & =\frac{k}{d_{\operatorname{LoS}} \sqrt{\eta^{\operatorname{LoS}}}} \sqrt{2 P_{\mathrm{d}} G\left(\beta_{\mathrm{d}}\right)} g_{\mathrm{d}}\left(t-\tau_{\mathrm{d}}\right) \\
& \times \cos \left(2 \pi f_{\mathrm{c}} t+\theta_{\mathrm{d}}\right),
\end{aligned}
$$

while the NLoS component is obtained by applying both the small- and large-scale fading along with the sensor antenna pattern as follows

$$
\begin{aligned}
Z_{\mathrm{NLoS}}(t) & =\sum_{m=1}^{M_{\mathrm{d}}} \alpha_{m} \frac{k}{d_{\mathrm{LoS}} \sqrt{\eta^{\mathrm{NLoS}}}} \sqrt{2 P_{\mathrm{d}} G\left(\beta_{m}\right)} g_{\mathrm{d}}\left(t-\tau_{\mathrm{d}}\right) \\
& \times \cos \left(2 \pi f_{\mathrm{c}} t+\theta_{\mathrm{d}}+\phi_{m}\right)
\end{aligned}
$$


As before, the composite SOI is computed by (16) and (17) as

$$
Z(t)=\left\{\begin{array}{ll}
Z_{\mathrm{LoS}}(t)+Z_{\mathrm{NLoS}}(t), & \text { with } \mathrm{P}_{\operatorname{LoS}}^{\mathrm{A}}\left(r_{\mathrm{d}}\right) \\
Z_{\mathrm{NLoS}}(t), & \text { with } 1-\mathrm{P}_{\mathrm{LoS}}^{\mathrm{A}}\left(r_{\mathrm{d}}\right)
\end{array},\right.
$$

where $\mathrm{P}_{\mathrm{LoS}}^{\mathrm{A}}\left(r_{\mathrm{d}}\right)$ is the probability of the respective LoS state given in (3). The notation used in (16) and (17) is as follows: $k=\frac{\mathrm{c}}{4 \pi f_{\mathrm{c}}}, P_{\mathrm{d}}$ is the transmit power of the drone, $g_{\mathrm{u}}(t)$ is the unit-energy signal waveform for the drone, $\tau_{d}$ is the random delay in the transmission of the drone with respect to the probe sensor, and $\theta_{\mathrm{d}}$ is the signal phase of the drone taking values in the range $[0,2 \pi)$. As we do not assume to have any knowledge of the drone signal waveform at the probe sensor, we assume that $\theta_{\mathrm{d}} \sim \mathcal{U}[0,2 \pi), \tau_{\mathrm{d}} \sim \mathcal{U}[0, T]$. This enables us to be inclusive of most existing RF infrastructures. We can thus project a realistic bound on the detection performance, by accounting for the respective performance losses, due to the lack of the said signal information.

We assume that both UE and the drone signals are narrowband, such that $f_{\mathrm{c}} \gg \frac{1}{T}$ with $T$ being the symbol duration at the probe sensor. For the sake of analytical tractability, we assume the general signal waveforms for the UEs and the drone to be a unit-energy step function:

$$
u(t)=\left\{\begin{array}{ll}
\frac{1}{\sqrt{T}}, & \text { for } 0 \leq t \leq T \\
0, & \text { otherwise }
\end{array} .\right.
$$

Lemma 1: The in-phase/quadrature (I/Q) decomposition of the composite received signal $R(t)$ is represented as $\boldsymbol{R}=\boldsymbol{Z}+\boldsymbol{Y}+\boldsymbol{N}$. The IQ components of the AWGN, $N_{\mathrm{I}}$ and $N_{\mathrm{Q}}$ (such that $\boldsymbol{N}=N_{\mathrm{l}}+N_{\mathrm{Q}}$ ) are i.i.d Gaussian random variables, each distributed as $\sim \mathcal{N}\left(0, N_{0} / 2\right)$ [31], [32], and the IQ-decomposition for the aggregate interference is given in (20), where $\rho_{i, \mathrm{u}}=\int_{0}^{T} g_{\mathrm{u}}\left(t-\tau_{i}\right) g_{\mathrm{u}}(t) \mathrm{d} t$ is the crosscorrelation between the transmit and receive waveforms for the $i$-th nearest UE, and $\left\{\rho_{i, u}\right\}$ is a sequence of i.i.d random variables with $\rho_{i, \mathrm{u}} \sim \mathcal{U}[0,1]$. Similarly, I/Q decomposition of the SOI is given in (21), where $\eta=\eta^{\mathrm{NLoS}} / \eta^{\mathrm{LoS}}$, and $\rho_{\mathrm{d}}=\int_{0}^{T} g_{\mathrm{d}}(t-\tau) g_{\mathrm{u}}(t) \mathrm{d} t$ is the respective cross-correlation and $\rho_{\mathrm{d}} \sim \mathcal{U}[0,1]$.

Proof: See Appendix A.

\section{Vi. Detection and Coverage Performance of the RF SENSING NETWORK}

In this section, we evaluate the detection performance of the sensing network. To this end, we first derive the distribution of the RSS of the composite received signal at the probe sensor, and then derive the probabilities of false alarm, detection and coverage.

\section{A. Statistical Distribution of RSS}

In order to derive the distribution of the RSS, we first describe the distribution of the I/Q components of the aggregate interference and the SOI in the following two theorems, and then present the overall distribution of the received signal.

Theorem 1: For a sufficiently large number of MPCs over the NLoS link (i.e., $M_{\mathrm{u}}$ ), a given value of the horizontal distance $R_{1, \mathrm{u}}$, a given value of the UE cross correlation coefficient $\rho_{1, \mathrm{u}}$, a given value of intended UE's signal phase $\theta_{1, \mathrm{u}}$ and a given value of the Stable random variable $V$ (defined below), the conditional distributions of the I-Q components $\left(\boldsymbol{Y}=Y_{\mathrm{I}}+j Y_{\mathrm{Q}}\right)$ of $Y$ are given as follows $Y_{\mathrm{I}} \sim \mathcal{N}\left(\mu_{\mathrm{I}, \mathrm{Y}}, \sigma_{\mathrm{Y}}^{2}\right)$ and $Y_{\mathrm{Q}} \sim \mathcal{N}\left(\mu_{\mathrm{Q}, \mathrm{Y}}, \sigma_{\mathrm{Y}}^{2}\right)$, where

$$
\mu_{\mathrm{I}, \mathrm{Y}}=\left\{\begin{array}{ll}
\frac{k \rho_{1, \mathrm{u}} \sqrt{P_{\mathrm{u}} G\left(\beta_{1, \mathrm{u}}\right)} \cos \left(\theta_{1, \mathrm{u}}\right)}{R_{1, \mathrm{u}}^{\gamma \mathrm{L}}}, & \text { with } \mathrm{P}_{\mathrm{LoS}}^{\top}\left(r_{1, \mathrm{u}}\right) \\
0, & \text { with } 1-\mathrm{P}_{\mathrm{LoS}}^{\top}\left(r_{1, \mathrm{u}}\right)
\end{array},\right.
$$

and

$$
\mu_{\mathrm{Q}, \mathrm{Y}}=\left\{\begin{array}{ll}
\frac{k \rho_{1, \mathrm{u}} \sqrt{P_{\mathrm{u}} G\left(\beta_{1, \mathrm{u}}\right)} \sin \left(\theta_{1, \mathrm{u}}\right)}{R_{1, \mathrm{u}}^{\gamma}}, & \text { with } \mathrm{P}_{\mathrm{LoS}}^{\top}\left(r_{1, \mathrm{u}}\right) \\
0, & \text { with } 1-\mathrm{P}_{\mathrm{LoS}}^{\top}\left(r_{1, \mathrm{u}}\right)
\end{array},\right.
$$

with the variance

$$
\sigma_{\mathrm{Y}}^{2}=\frac{4 V k^{\gamma_{1}} P_{\mathrm{u}}}{\pi^{\left(\gamma_{1}-1\right)}}\left[k_{\mathrm{u}} \lambda \mathrm{C}_{\frac{4}{\gamma_{1}}}^{-1} \frac{2}{\gamma_{\mathrm{I}}+2} \Gamma\left(\frac{\gamma_{\mathrm{I}}+8}{2 \gamma_{\mathrm{l}}}\right) h\left(\beta_{3 \mathrm{~dB}}\right)\right]^{\frac{\gamma_{1}}{2}},
$$

where $C_{\frac{4}{\gamma_{1}}}$ is a parameter based on the particular value of $\gamma_{1}$, $h\left(\beta_{3 \mathrm{~dB}}\right)$ is the function given as

$$
\begin{aligned}
& h\left(\beta_{3 \mathrm{~dB}}\right)=\beta_{3 \mathrm{~dB}}\left(\frac{e^{0.23 G_{\mathrm{P}, \text { max }}}}{\sqrt{0.23 \pi}} \operatorname{erf}\left(\sqrt{\frac{0.23 A_{\mathrm{m}}}{12}}\right)\right) \\
& -\beta_{3 \mathrm{~dB}}\left(\frac{2}{\pi} \sqrt{\frac{A_{\mathrm{m}}}{12}} e^{G_{\mathrm{P}, \text { max }}-A_{\mathrm{m}}}\right)+e^{G_{\mathrm{P}, \text { max }}-A_{\mathrm{m}}},
\end{aligned}
$$

and $V$ is the random variable following a Stable distribution, denoted $\mathcal{S}\left(\alpha_{\mathrm{V}}, \beta_{\mathrm{V}}, \gamma_{\mathrm{V}}\right)$, with the stability parameter $\alpha_{\mathrm{V}}=\frac{2}{\gamma_{1}}$, the skewness parameter $\beta_{\mathrm{V}}=1$, and the scale (dispersion) parameter $\gamma_{V}=\cos \left(\frac{\pi}{\gamma_{1}}\right)$ [33]. The choice of isotropic antenna at the probe sensor results in $h\left(\beta_{3 \mathrm{~dB}}\right)=1$.

Proof: See Appendix B.

Theorem 2: For a sufficiently large number of MPCs over the NLoS link (i.e., $M_{\mathrm{d}}$ ), a given value of the horizontal distance $R_{\mathrm{d}}$, a given value of the drone correlation coefficient $\rho_{\mathrm{d}}$, a given value of the random phase $\theta_{\mathrm{d}}$, and a given value of the Stable random variable $V$ the conditional distributions of the I-Q components $\left(\boldsymbol{Z}=Z_{1}+j Z_{\mathrm{Q}}\right)$ of $Z$ are given as follows $Z_{\mathrm{I}} \sim \mathcal{N}\left(\mu_{\mathrm{l}, Z}, \sigma_{Z}^{2}\right)$ and $Z_{\mathrm{Q}} \sim \mathcal{N}\left(\mu_{\mathrm{Q}, Z}, \sigma_{Z}^{2}\right)$, where

$$
\mu_{\mathrm{l}, \mathrm{Z}}=\left\{\begin{array}{ll}
\frac{k \rho_{\mathrm{d}} \sqrt{P_{\mathrm{d}} G\left(\beta_{\mathrm{d}}\right)} \cos \left(\theta_{\mathrm{d}}\right)}{d_{\mathrm{LoS}} \sqrt{\eta^{\operatorname{LoS}}},}, & \text { with } \mathrm{P}_{\mathrm{LoS}}^{\mathrm{A}}\left(r_{\mathrm{d}}\right) \\
0, & \text { with } 1-\mathrm{P}_{\text {LoS }}^{\mathrm{A}}\left(r_{\mathrm{d}}\right)
\end{array},\right.
$$

and

$$
\mu_{\mathrm{Q}, \mathrm{Z}}=\left\{\begin{array}{ll}
\frac{k \rho_{\mathrm{d}} \sqrt{P_{\mathrm{d}} G\left(\beta_{\mathrm{d}}\right)} \sin \left(\theta_{\mathrm{d}}\right)}{d_{\mathrm{LoS}} \sqrt{\eta^{\operatorname{LoS}}},}, & \text { with } \mathrm{P}_{\mathrm{LoS}}^{\mathrm{A}}\left(r_{\mathrm{d}}\right) \\
0, & \text { with } 1-\mathrm{P}_{\text {LoS }}^{\mathrm{A}}\left(r_{\mathrm{d}}\right)
\end{array},\right.
$$

with the variance

$$
\sigma_{\mathrm{Z}}^{2}=\frac{2 k^{2} \rho_{\mathrm{d}}^{2} P_{\mathrm{d}} h\left(\beta_{3 \mathrm{~dB}}\right)}{d_{\mathrm{LoS}}^{2} \eta^{\mathrm{NLoS}}} .
$$




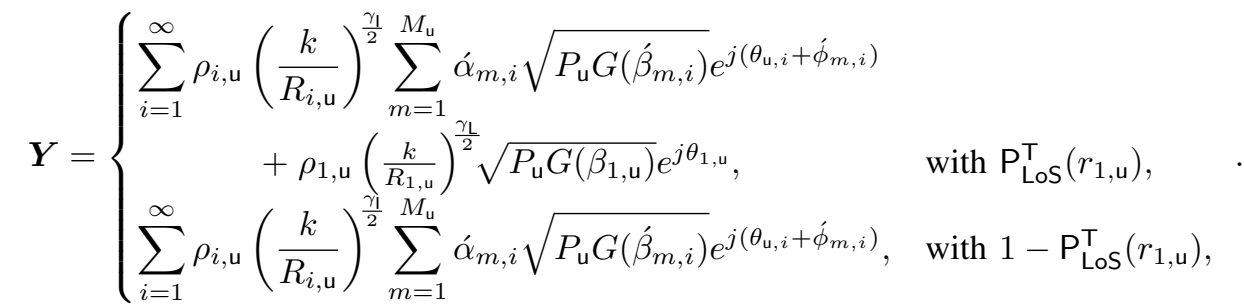

$$
\begin{aligned}
& \boldsymbol{Z}=\left\{\begin{array}{ll}
\frac{k \rho_{\mathrm{d}} e^{j \theta_{\mathrm{d}}}}{d_{\mathrm{LoS}} \sqrt{\eta^{\mathrm{NLoS}}}}\left[\sum_{m=1}^{M_{\mathrm{d}}} \alpha_{m} \sqrt{P_{\mathrm{d}} G\left(\beta_{\mathrm{m}}\right)} e^{j \phi_{m}}+\sqrt{\eta P_{\mathrm{d}} G\left(\beta_{\mathrm{d}}\right)}\right], & \text { with } \mathrm{P}_{\mathrm{LoS}}^{\mathrm{A}}\left(r_{\mathrm{d}}\right), \\
\frac{k \rho_{\mathrm{d}} e^{j \theta_{\mathrm{d}}}}{d_{\mathrm{LoS}} \sqrt{\eta^{\mathrm{NLOS}}}} \sum_{m=1}^{M_{\mathrm{d}}} \alpha_{m} \sqrt{P_{\mathrm{d}} G\left(\beta_{\mathrm{m}}\right)} e^{j \phi_{m}}, & \text { with } 1-\mathrm{P}_{\mathrm{LoS}}^{\mathrm{A}}\left(r_{\mathrm{d}}\right),
\end{array} .\right.
\end{aligned}
$$

Proof: See Appendix C.

Let $R_{\mathrm{I}}$ and $R_{\mathrm{Q}}$ be the in-phase and the quadrature components of the I/Q decomposition of the received signal such that $\boldsymbol{R}=R_{1}+j R_{\mathrm{Q}}$. The RSS is therefore given in terms of in-phase and quadrature components as follows:

$$
R_{\mathrm{s}}=R_{\mathrm{I}}^{2}+R_{\mathrm{Q}}^{2}
$$

Using the distributions of the I/Q decomposition of the aggregate interference and SOI given in Theorem 1 and Theorem 2, we characterize the distribution of the RSS under the hypotheses $\mathcal{H}_{0}$ and $\mathcal{H}_{1}$ defined in (10) and (11), respectively, in the following theorems.

Theorem 3: For a given value of the horizontal distance $R_{1, \mathrm{u}}$, a given value of the UE correlation coefficient $\rho_{1, \mathrm{u}}$, and a given value of the Stable random variable $V$, the conditional distributions of the RSS under $\mathcal{H}_{0}$ can be described as a scaled non-central Chi-squared distribution of degree 2 and non-centrality parameter $\frac{\lambda_{\text {nc }}}{\sigma_{0}^{2}}$, i.e., $R_{\mathrm{s}} \sim \sigma_{0} \chi_{2}^{\prime 2}\left(\frac{\lambda_{\text {nc }}}{\sigma_{0}^{2}}\right)$, where

$$
\begin{aligned}
\sigma_{0}^{2} & =\sigma_{\mathrm{Y}}^{2}+\frac{N_{0}}{2}, \\
\lambda_{\mathrm{nc}} & = \begin{cases}\frac{k^{2} \rho_{1, \mathrm{u}}^{2} P_{\mathrm{u}} G\left(\beta_{1, \mathrm{u}}\right)}{R_{1, \mathrm{u}}^{\gamma \mathrm{L}}}, & \text { with } \mathrm{P}_{\mathrm{LoS}}^{\top}\left(r_{1, \mathrm{u}}\right) \\
0, & \text { with } 1-\mathrm{P}_{\mathrm{LoS}}^{\top}\left(r_{1, \mathrm{u}}\right)\end{cases}
\end{aligned}
$$

and $\sigma_{\mathrm{Y}}^{2}$ is given in (24). Note that when $\lambda_{\mathrm{nc}}=0$ (i.e., along with the NLoS channel state), the distribution of $R_{\mathrm{s}}$ reduces to a scaled Chi-squared distribution.

Proof: See Appendix D.

Theorem 4: For a given value of the horizontal intended UE distance $R_{1, \mathrm{u}}$, the horizontal drone distance $R_{\mathrm{d}}$, a given value of the drone correlation coefficient $\rho_{\mathrm{d}}$, a given value of the UE correlation coefficient $\rho_{1, \mathrm{u}}$, and a given value of the Stable random variable $V$, the conditional distributions of the RSS under $\mathcal{H}_{1}$ can be described as a scaled non-central Chisquared distribution of degree 2 and non-centrality parameter $\frac{\lambda_{\text {nc }}}{\sigma_{1}^{2}}$, i.e., $R_{\mathrm{s}} \sim \sigma_{1} \chi_{2}^{\prime 2}\left(\frac{\lambda_{\text {nc }}}{\sigma_{1}^{2}}\right)$, where

$$
\sigma_{1}^{2}=\sigma_{\mathrm{Z}}^{2}+\sigma_{\mathrm{Y}}^{2}+\frac{N_{0}}{2},
$$

and

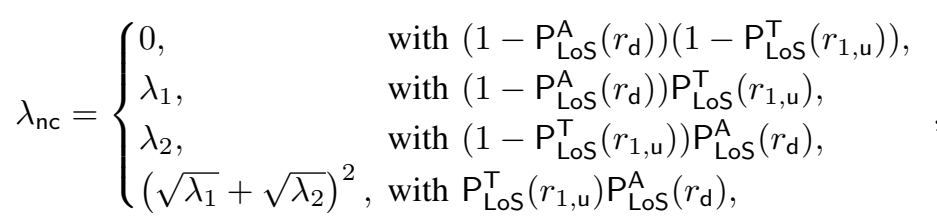

where $\sigma_{\mathrm{Z}}^{2}$ is given in (28), and

$$
\lambda_{1}=\frac{k^{2} \rho_{1, \mathrm{u}}^{2} P_{\mathrm{u}} G\left(\beta_{1, \mathrm{u}}\right)}{R_{1, \mathrm{u}}^{\gamma_{\mathrm{L}}}}, \quad \lambda_{2}=\frac{k^{2} \rho_{\mathrm{d}}^{2} P_{\mathrm{d}} G\left(\beta_{\mathrm{d}}\right)}{\eta^{\mathrm{LoS}} d_{\mathrm{LoS}}^{2}} .
$$

Proof: See Appendix E.

\section{B. Performance Evaluation}

We now investigate the detection performance by deriving the probability of false alarm, referred to as $P_{\mathrm{FA}}$, the conditional probability of detection for a given value of $r_{\mathrm{d}}$, referred to as $P_{\mathrm{D}}\left(r_{\mathrm{d}}\right)$, and the network wide detection coverage probability referred to as $P_{\mathrm{D}, \mathrm{Avg}}$. We decide on $\mathcal{H}_{1}$ when the RSS measurement exceeds a fixed threshold $\gamma_{\mathrm{th}}$, and pick $\mathcal{H}_{0}$ otherwise. $P_{\mathrm{FA}}$ and $P_{\mathrm{D}}\left(r_{\mathrm{d}}\right)$ can therefore be derived using the complimentary cumulative distribution functions $(\mathrm{CCDFs}) \bar{F}_{R_{\mathrm{s}}}\left(\gamma_{\mathrm{th}} ; \mathcal{H}_{0}\right)=\operatorname{Pr}\left(R_{\mathrm{s}} \geq \gamma_{\mathrm{th}} ; \mathcal{H}_{0}\right)$ and $\bar{F}_{R_{\mathrm{s}} \mid R_{\mathrm{d}}}\left(\gamma_{\mathrm{th}} \mid r_{\mathrm{d}} ; \mathcal{H}_{1}\right)=\operatorname{Pr}\left(R_{\mathrm{s}} \geq \gamma_{\mathrm{th}} \mid r_{\mathrm{d}} ; \mathcal{H}_{1}\right)$, respectively.

Theorem 5: The probability of false alarm, $P_{\mathrm{FA}}$, at the probe sensor for the drone detection problem described in Section III is given in (34), where $\sigma_{0}^{2}$ and $\lambda_{1}$ are given in (30) and (33), respectively, and $Q_{\mathrm{M}}(\cdot)$ is the Marcum Q-function [34].

Note that the distribution of the aggregate interference, as stated by (22), (23), and (24), does not depend on the location in the stationary HPPP $\Phi_{\mathrm{B}}$ [25]. Moreover, the distribution of the interference is not impacted by the location of the target drone either. Therefore the probability of false alarm at all sensor locations in the network $\Phi_{\mathrm{B}}$, for all possible locations of the target drone, has the same value and it is represented by (35).

Proof: See Appendix D.

On the contrary, the distribution of the RSS, as stated by (26), (27), and (28), in the presence of a target drone is dependent on the link distance between the target drone and the probe sensor. Therefore considering the horizontal distance $\left(r_{\mathrm{d}}\right)$ between the drone and the sensor to be a constant, enables 


$$
\begin{aligned}
P_{\mathrm{FA}}= & \mathbb{E}_{V}\left[\int _ { 0 } ^ { \infty } \int _ { 0 } ^ { 1 } 2 \pi k _ { \mathrm { u } } \lambda r _ { 1 , \mathrm { u } } \operatorname { e x p } ( - \lambda \pi r _ { 1 , \mathrm { u } } ^ { 2 } ) \left(\mathrm{P}_{\mathrm{LoS}}^{\top}\left(r_{1, \mathrm{u}}\right) Q_{\mathrm{M}}\left(\sqrt{\frac{\lambda_{1}}{\sigma_{0}^{2}}}, \sqrt{\frac{\gamma_{\mathrm{th}}}{\sigma_{0}^{2}}}\right)\right.\right. \\
& \left.\left.+\mathrm{P}_{\mathrm{NLoS}}^{\top}\left(r_{1, \mathrm{u}}\right) \exp \left(-\frac{\gamma_{\mathrm{th}}}{2 \sigma_{0}^{2}}\right)\right) \mathrm{d} \rho_{1, \mathrm{u}} \mathrm{d} r_{1, \mathrm{u}}\right] . \\
P_{\mathrm{D}}\left(r_{\mathrm{d}}\right)= & \mathbb{E}_{V}\left[\int _ { 0 } ^ { \infty } \int _ { 0 } ^ { 1 } \int _ { 0 } ^ { 1 } 2 \pi k _ { \mathrm { u } } \lambda r _ { 1 , \mathrm { u } } \operatorname { e x p } ( - \lambda \pi r _ { 1 , \mathrm { u } } ^ { 2 } ) \left(\mathrm{P}_{\mathrm{LoS}}^{\top}\left(r_{1, \mathrm{u}}\right) \mathrm{P}_{\mathrm{NLoS}}^{\mathrm{A}}\left(r_{\mathrm{d}}\right) Q_{\mathrm{M}}\left(\sqrt{\frac{\lambda_{1}}{\sigma_{1}^{2}}}, \sqrt{\frac{\gamma_{\mathrm{th}}}{\sigma_{1}^{2}}}\right)\right.\right. \\
+ & \mathrm{P}_{\mathrm{NLoS}}^{\top}\left(r_{1, \mathrm{u}}\right) \mathrm{P}_{\mathrm{LoS}}^{\mathrm{A}}\left(r_{\mathrm{d}}\right) Q_{\mathrm{M}}\left(\sqrt{\frac{\lambda_{2}}{\sigma_{1}^{2}}}, \sqrt{\frac{\gamma_{\mathrm{th}}}{\sigma_{1}^{2}}}\right)+\mathrm{P}_{\mathrm{LoS}}^{\top}\left(r_{1, \mathrm{u}}\right) \mathrm{P}_{\mathrm{LoS}}^{\mathrm{A}}\left(r_{\mathrm{d}}\right) Q_{\mathrm{M}}\left(\sqrt{\frac{\lambda_{3}}{\sigma_{1}^{2}}}, \sqrt{\frac{\gamma_{\mathrm{th}}}{\sigma_{1}^{2}}}\right) \\
+ & \left.\left.\mathrm{P}_{\mathrm{NLoS}}^{\top}\left(r_{1, \mathrm{u}}\right) \mathrm{P}_{\mathrm{NLoS}}^{\mathrm{A}}\left(r_{\mathrm{d}}\right) \exp \left(-\frac{\gamma_{\mathrm{th}}}{2 \sigma_{1}^{2}}\right)\right) \mathrm{d} \rho_{\mathrm{d}} \mathrm{d} \rho_{1, \mathrm{u}} \mathrm{d} r_{1, \mathrm{u}}\right] .
\end{aligned}
$$

us to evaluate the impact of the interferer density, the drone altitude, and the RF configuration (i.e. the beam tilt and the HPBW) on the detection probability, $P_{\mathrm{D}}\left(r_{\mathrm{d}}\right)$, regardless of the sensor density. Thus $P_{\mathrm{D}}\left(r_{\mathrm{d}}\right)$ indicates the detection probability, at the probe sensor (located at the origin), due to a given set of drone locations, $\left\{\left(x, y, h_{\mathrm{d}}\right) \in \mathbb{R}^{3}: x^{2}+y^{2}=r_{\mathrm{d}}{ }^{2}\right\}$, and is given in the following theorem.

Theorem 6: The conditional probability of detection , $P_{\mathrm{D}}\left(r_{\mathrm{d}}\right)$, at the probe sensor that is horizontally $r_{\mathrm{d}}$ units away from the target drone is given in (35), where $\sigma_{1}^{2}$ and $\lambda_{2}$ are given in (31) and (33), respectively, and $\lambda_{3}=\left(\sqrt{\lambda_{1}}+\sqrt{\lambda_{2}}\right)^{2}$. Since the closed form PDF for the stable random variable $V$ is not available, the expectation with respect to $V, \mathbb{E}_{V}[$.$] is$ computed by averaging the respective function of $V$ over a large number of samples drawn from the given distribution of $V$.

Proof: See Appendix E.

In order to evaluate the detection coverage probability achieved by the sensor network in the ROI, we consider detection by the $n^{\text {th }}$-nearest sensor, and the distribution of the random horizontal distance $\left(R_{\mathrm{d}}\right)$ between the target drone and the probe sensor is then given by (2). Following the notion of single coverage [5], [25], a certain drone location $\left(x, y, h_{\mathrm{d}}\right)$ in the ROI is said to be covered if the drone is successfully detected by at least one sensor in the network, and the coverage probability is defined as the fraction of the ROI that at least has single-coverage. Thus the coverage probability, $P_{\mathrm{D}, \mathrm{Avg}}$, achieved by the sensor network, $\Phi_{\mathrm{B}}$, is given in the following theorem.

Theorem 7: The detection coverage probability, $P_{\mathrm{D} \text {,avg }}$, can hence be obtained by taking expectation of the conditional probability of detection, $P_{\mathrm{D}}\left(r_{\mathrm{d}}\right)$ in (35), with respect to the random variable $R_{\mathrm{d}}$, over the all possible values of $r_{\mathrm{d}}$ as follows

$$
P_{\mathrm{D}, \text { avg }}=\int_{0}^{\infty} \frac{2(\pi \lambda)^{n}}{\Gamma(n)} r_{\mathrm{d}}^{2 n-1} \exp \left(-r_{\mathrm{d}}^{2}\right) P_{\mathrm{D}}\left(r_{\mathrm{d}}\right) \mathrm{d} r_{\mathrm{d}},
$$

which characterizes the detection performance regardless of particular location (i.e. a particular value of $R_{\mathrm{d}}$ ) of the target drone in the network.

Proof: See Appendix F.

\section{NUMERICAL ANALYSIS}

In this section, we numerically analyze the impact of various network, propagation and RF parameters on the detection performance of the proposed detector.

\section{A. Overview of Key Expressions}

Before we provide our numerical results, in this section we would like to summarize some of the key expressions derived in the earlier sections and comment on their significance. In Section IV, equations (22) through (25) characterize the mean $\left(\mu_{1, Y}, \mu_{\mathrm{Q}, \mathrm{Y}}\right)$ and variance $\left(\sigma_{\mathrm{Y}}^{2}\right)$ of the I-Q components of the total interference signal, $\boldsymbol{Y}$, at the probe BS. Equations (26) through (28) characterize the mean $\left(\mu_{1, Z}, \mu_{\mathrm{Q}, \mathrm{Z}}\right)$ and the variance $\left(\sigma_{\mathrm{Z}}^{2}\right)$ of the I-Q components of the drone signal, $Z$, at the probe BS. The reason for considering these equations as crucial, is that the mean and the variance terms are expressed in terms of the node density $(\lambda)$, antenna pattern parameters ( $\beta_{\text {tilt }}$, and $\left.\beta_{3 \mathrm{~dB}}\right)$, and the $\mathrm{A} 2 \mathrm{G}$ and terrestrial propagation parameters. Thus, these equations are crucial to understanding how the LoS and NLoS energies in these signals are dependent on these factors, which in turn decides the distribution of the received signal strength (RSS) (as discussed in Section V, and represented by equations (30) through (33)) at the BS, and the final performance metrics such as $P_{\mathrm{D}}$ and $P_{\mathrm{FA}}$, which are essentially the complementary cumulative distribution functions (CCDF) of the RSS, under the alternative and the null hypothesis.

Finally, equations (34)-(36) characterize the immediate performance metrics of interest, i.e. the conditional detection probability, $P_{\mathrm{D}}\left(r_{\mathrm{d}}\right)$, the probability of the false alarm, $P_{\mathrm{FA}}$, and the detection coverage probability, $P_{\mathrm{D}, \text { Avg }}$, and thus they are crucial to gaining understanding of the numerical results.

\section{B. Numerical Results}

In particular for the case of the isotropic antenna pattern, the conditional detection performance is evaluated in Section VII-C by plotting $P_{\mathrm{D}}\left(r_{\mathrm{d}}\right)$ in (35) against the respective $P_{\mathrm{FA}}$ (i.e., the receiver operating characteristic (ROC)), whereas the detection coverage performance is depicted by plotting $P_{\mathrm{D} \text {,avg }}$ in (36) for a fixed desired value of $P_{\mathrm{FA}}=\alpha_{\mathrm{FA}}$. For the 


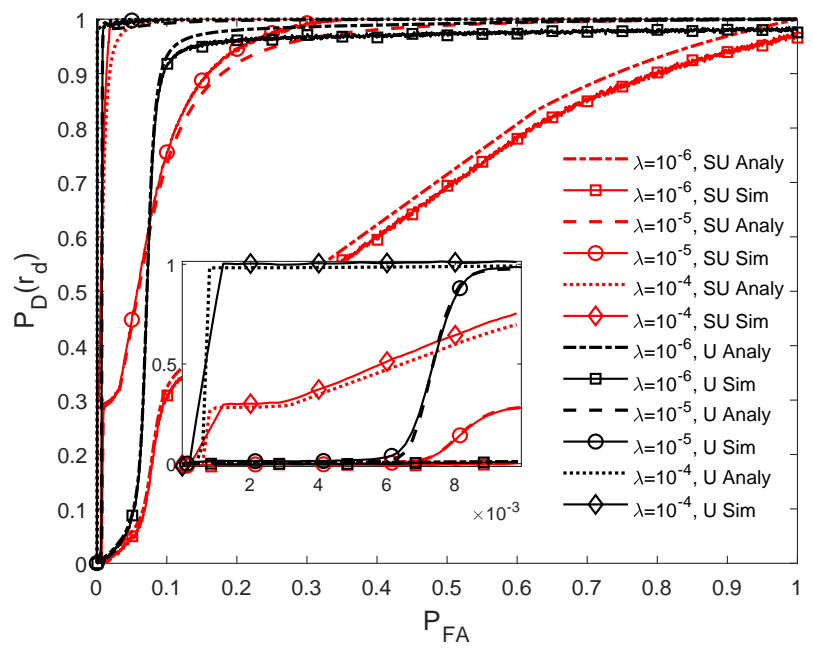

(a) NLoS PLE, $\gamma_{1}=4$.

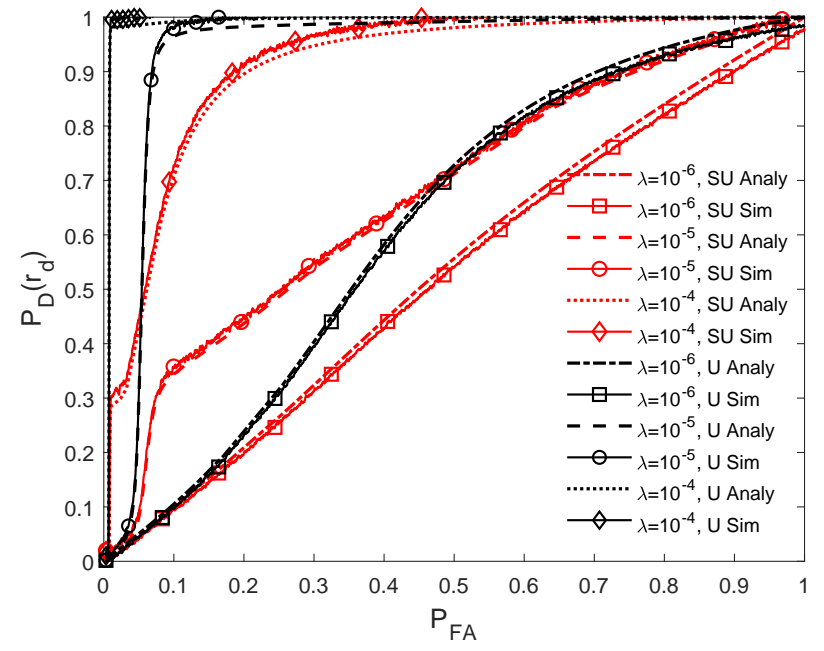

(b) NLoS PLE, $\gamma_{1}=3.5$.

Fig. 6: Single sensor ROCs for varying node densities $\left(h_{\mathrm{d}}=300 \mathrm{~m}, r_{\mathrm{d}}=923 \mathrm{~m}\right.$, Isotropic).

directional antenna pattern, the conditional (see Section VII-D) and the detection coverage (see Section VII-E) performances are plotted in pairs, i.e., $\left(P_{\mathrm{D}}\left(r_{\mathrm{d}}\right), P_{\mathrm{FA}}\right)$ and $\left(P_{\mathrm{D} \text {,avg }}, P_{\mathrm{FA}}\right)$, respectively, as functions of beam tilt, HPBW, drone altitude, and node density.

We assume a fully-loaded network with $k_{\mathrm{u}}=1$ in the interference-limited regime so that the AWGN is negligible compared to the interference power, i.e., $\sigma_{Y}^{2} \gg \frac{N_{0}}{2}$ so that $\sigma_{0}^{2}=\sigma_{Y}^{2}$. We further assume $P_{\mathrm{u}}=P_{\mathrm{d}}=20 \mathrm{dBm}, f_{\mathrm{c}}=3.5$ $\mathrm{GHz}, h_{\mathrm{s}}=35 \mathrm{~m}, h_{\mathrm{ue}}=0 \mathrm{~m}, G_{\mathrm{P}, \max }=17 \mathrm{~dB}$, and $A_{\mathrm{m}}=20$ $\mathrm{dB}$ [23]. The terrestrial channel is identified with the PLE $\gamma_{\mathrm{I}} \in[2.13,4.89]$ for the NLoS links [35] and $\gamma_{\mathrm{L}}=2$ for the LoS links. For this work, we consider suburban and urban settings for the A2G channel which are characterized by (3) and (4), with the following set of parameters: $(a, b)=(4.88,0.429)$, $\left(\eta^{\mathrm{LoS}}, \eta^{\mathrm{NLoS}}\right)=(0.1,21) \mathrm{dB}$ for the suburban setting, and $(a, b)=(9.6117,0.1581),\left(\eta^{\text {LoS }}, \eta^{\mathrm{NLOS}}\right)=(1,20) \mathrm{dB}$ for the urban setting [26].

\section{Sensors with Isotropic Antenna Patterns}

Fig. 6a and Fig. 6b show the ROCs for the isotropic antenna pattern and two different PLEs where the UE density $\lambda$ increases from $10^{-6} \mathrm{~m}^{-2}$ to $10^{-4} \mathrm{~m}^{-2}$. Regardless of the PLEs, the conditional detection performance $P_{\mathrm{D}}\left(r_{\mathrm{d}}\right)$ for a given drone location $\left(h_{\mathrm{d}}=300 \mathrm{~m}, \beta_{\mathrm{d}}=18^{\circ}\right.$ i.e. $\left.r_{\mathrm{d}}=923 \mathrm{~m}\right)$ drops for all values of $P_{\mathrm{FA}}$, as the UE density increases. This is because any increase in $\lambda$ also increases the dispersion $V \gamma_{\mathrm{G}}$ of the aggregate interference. For a given $\lambda$ and $P_{\mathrm{F} A}$, the $P_{\mathrm{D}}\left(r_{\mathrm{d}}\right)$ in a suburban area is higher than that in an urban area, which is due to the higher probability of $\operatorname{LoS}$ and the lower $A 2 \mathrm{G}$ path loss experienced in a suburban area. By comparing Fig. 6a and Fig. 6b, we see that for a fixed $P_{\mathrm{F} A}$ and $\lambda$, the $P_{\mathrm{D}}\left(r_{\mathrm{d}}\right)$ for $\gamma_{1}=3.5$ is significantly lower than that for $\gamma_{1}=4$. This is due to the fact that the dispersion of the aggregate interference increases as the PLE $\gamma_{1}$ for the interfering links decreases $\left(\gamma_{G} \propto \lambda^{\frac{\gamma_{1}}{2}}\right)$. Fig. 7a demonstrates the impact of the drone altitude on the conditional detection performance of the probe sensor for $\gamma_{\mathrm{l}}=4$. We observe that for $r_{\mathrm{d}}=923 \mathrm{~m}$, as $h_{\mathrm{d}}$ increases from $300 \mathrm{~m}$ to $600 \mathrm{~m}, P_{\mathrm{D}}\left(r_{\mathrm{d}}\right)$ drops for all values of $P_{\mathrm{F} A}$. This is due to the fact that the increased $\mathrm{A} 2 \mathrm{G}$ link distance $d_{\text {LoS }}$ causes a drop in the SIR of the SOI.

Next, we use Fig. $7 b$ to illustrate the impact of the node density, $\lambda$ on the detection coverage performance. For a given value of $P_{\mathrm{FA}}, P_{\mathrm{D} \text {,avg }}$ changes in a non-monotonic pattern with respect to $\lambda$. When $\lambda$ increases, the reduced average A2G link distance and the increased LoS dominance increase the average SIR of the SOI causing the $P_{\mathrm{D} \text {,avg }}$ to increase. However the increased $\lambda$ also increases the aggregate interference which in turn reduces the $P_{\mathrm{D} \text {,avg }}$ for a fixed $P_{\mathrm{FA}}$. Thus we find that there exists a critical node density $\lambda_{c}$ that optimizes the detection coverage performance. We note that $\lambda_{c}$ in both suburban and urban environments increase as the required $P_{\mathrm{F} A}$ decreases from 0.0025 to 0.001 . We also observe that the value of $\lambda_{c}$ for a fixed value of $P_{\mathrm{F} A}$ in suburban area is lower than that in an urban area.

For all the plots in Fig. and Fig. we include the results obtained by both the theoretical analysis presented in this paper, and a simulation based investigation as well. While the curves with the markers represent the theoretical analysis, the line curves illustrate the results obtained from the simulation. We note that the theoretical analysis tracks the simulation results quite closely. Lastly the process of the simulation is the same as that proposed in papers such as [36]-[39], where we consider a $1 \mathrm{~km} \times 1 \mathrm{~km}$ system area, and randomly drop BSs and UEs at 2D coordinates, where each coordinate is generated according to a uniform distribution $x \sim \mathcal{U}[-500 \mathrm{~m}, 500 \mathrm{~m}]$, and $y \sim \mathcal{U}[-500 \mathrm{~m}, 500 \mathrm{~m}]$, with total number of the BSs and the UEs in the system area being $\lambda \mathrm{m}^{-2} \times\left(10^{6} \mathrm{~m}^{2}\right)$, and $k_{\mathrm{u}} \lambda \mathrm{m}^{-2} \times\left(10^{6} \mathrm{~m}^{2}\right)$, respectively. We then fix the drone location $\left(x_{\mathrm{d}}, y_{\mathrm{d}}, h_{\mathrm{d}}\right)$, such that $r_{\mathrm{d}}^{2}=x_{\mathrm{d}}^{2}+y_{\mathrm{d}}^{2} \leq(923)^{2}$, pick one of the nearest BSs to the said drone location, and compute the received signal strength at the chosen BS for the two cases, i.e. with and without a contribution from the drone signal, and plot the null and alternative empirical cumulative distributions of the received signal strength in these two cases to obtain the probability of detection, $P_{\mathrm{D}}\left(r_{\mathrm{d}}\right)$, and 


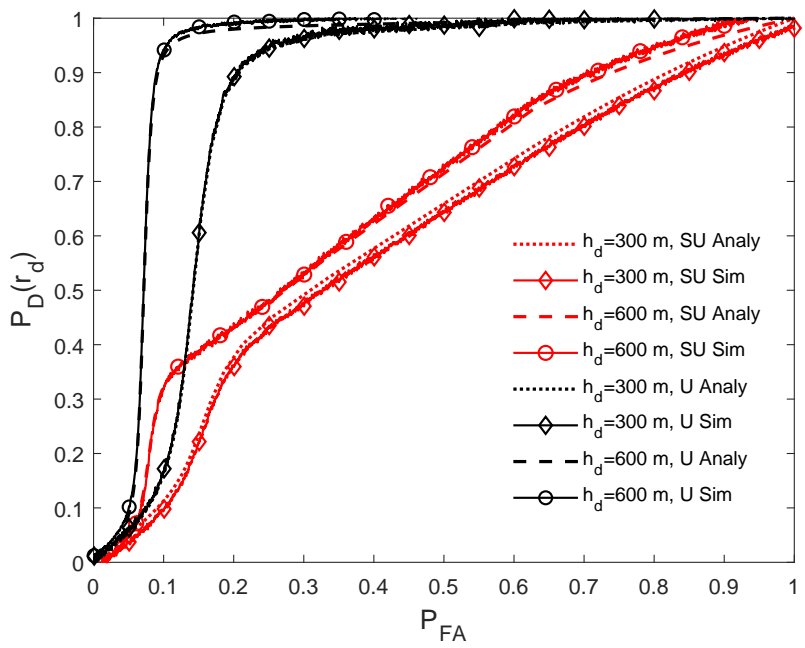

(a) Impact of $h_{\mathrm{d}}$ on ROC for $r_{\mathrm{d}}=923 \mathrm{~m}, \lambda=10^{-4} \mathrm{~m}^{-2}$.

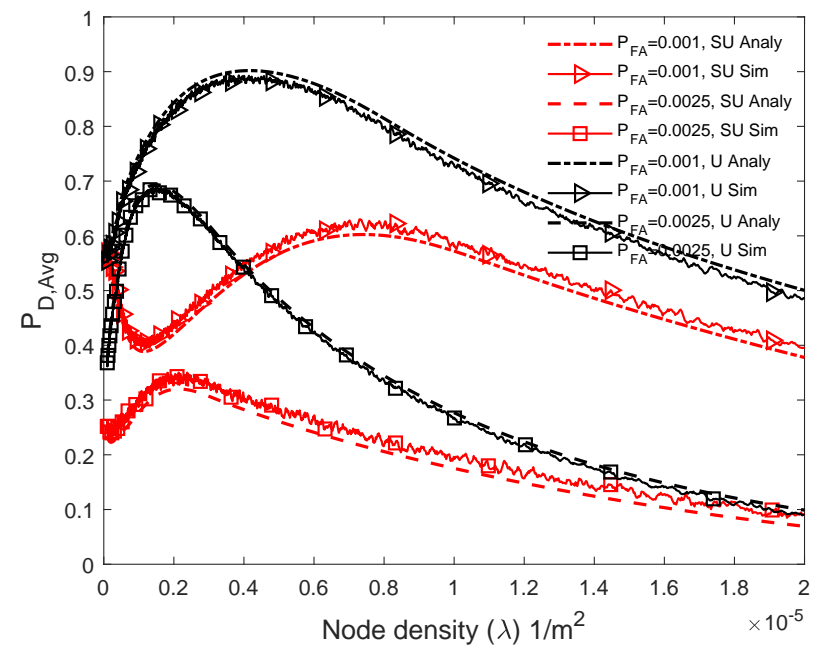

(b) Impact of $\lambda$ on $P_{\mathrm{D} \text {, avg }}$ for $h_{\mathrm{d}}=300 \mathrm{~m}$.

Fig. 7: Impact of $h$ and $\lambda$ on the detection performance $\left(\gamma_{I}=4\right.$, 'Isotropic').

the probability of false alarm, $P_{\mathrm{FA}}$, respectively. The coverage probability of detection, $P_{\mathrm{D} \text {, avg }}$, is obtained by generating the $2 \mathrm{D}$ coordinates of the drone location $\left(x_{\mathrm{d}}, y_{\mathrm{d}}\right)$ randomly according to the uniform distributions: $x_{\mathrm{d}} \sim \mathcal{U}[-500 \mathrm{~m}, 500 \mathrm{~m}]$, and $y_{\mathrm{d}} \sim \mathcal{U}[-500 \mathrm{~m}, 500 \mathrm{~m}]$, computing the probability of detection for each location of the drone, and then taking the average of the probability detection for all the drone locations.

\section{Sensors with Directional Antenna Patterns: Conditional Detection Performance}

In this section we discuss the impacts of the beam tilt, the HPWB, and the A2G propagation on $P_{\mathrm{D}}\left(r_{\mathrm{d}}\right)$ and $P_{\mathrm{FA}}$, and use the key ideas about probability of alignment described by (7) and (8) in Section. IV, to explain the variation in $P_{\mathrm{D}}\left(r_{\mathrm{d}}\right)$ and $P_{\mathrm{FA}}$ with respect to the said parameters. While the lack of alignment between the drone and the probe sensor is characterized by $\mathbb{P}(B)=0$, the receive gain of the LoS SOI and the LoS interference signal are monotonically increasing with respect to $\mathbb{P}(B)$ and $\mathbb{P}(A)$, respectively. Fig. 8a and Fig. $8 \mathrm{~b}$ together depict the impact of the antenna pattern alignment between the drone and the probe sensor, on the conditional detection performance. Both figures employ the same antenna pattern configuration: $\beta_{\text {tilt }} \in\left[-30^{\circ}, 30^{\circ}\right]$ and $\beta_{3 \mathrm{~dB}} \in\left\{8^{\circ}, 12^{\circ}\right\}$, but Fig. 8a considers a drone elevation angle $\left(\beta_{\mathrm{d}}=85^{\circ}, h_{\mathrm{d}}=100 \mathrm{~m}\right)$ that remains outside the drone detection zone $\left(\beta_{\mathrm{d}} \geq \beta_{\mathrm{ub}}\right)$ of the probe sensor for all $\beta_{\mathrm{tilt}}$ and all $\beta_{3 \mathrm{~dB}}$. Due to this lack of alignment the LoS drone signal experiences a very low receive gain of $-20 \mathrm{~dB}$ for all beam tilts, leading to very a low SOI and and a very poor $P_{\mathrm{D}}\left(r_{\mathrm{d}}\right)$. On the other hand, Fig. $8 \mathrm{~b}$ demonstrates the improved detection performance for a drone location $\left(\beta_{\mathrm{d}}=15^{\circ}, h_{\mathrm{d}}=100 \mathrm{~m}\right)$ that is within the detection zone for all values of $\beta_{3 \mathrm{~dB}}$ and a subrange of $\beta_{\text {tilt. }}$.

1) Impact of Antenna Tilt: Fig. 8a shows us that when $\mathbb{P}(B)=0$, there exist critical beam tilts, respectively $\beta_{\text {tilt, } \mathrm{P}_{\mathrm{D}}}^{\prime}$ and $\beta_{\text {tilt, } P_{\mathrm{D}}}^{\prime}$, that maximize $P_{\mathrm{D}}\left(r_{\mathrm{d}}\right)$ and $P_{\mathrm{FA}}$. This is because, as $\beta_{\text {tilt }}$ increases and the interference zone moves away from the origin, $\mathbb{P}(A)$ keeps increasing until the interference zone moves too far away beyond the intervals of $R_{1, \mathrm{u}}$ where the probability density $f_{R_{1, \mathrm{u}}}\left(r_{1, \mathrm{u}}\right)$ is high. This in turn increases the respective receive gain of the LoS interference signal, $Y_{1, \mathrm{~L}}(t)$. Since the increase in the receive gain is much stronger than the increased path loss and the decreased probability of LoS caused by the outward movement of the interference zone, in the absence of the drone-sensor alignment, the RSS of the received signal is mainly governed by $\mathbb{P}(A)$. Thus, similar to $\mathbb{P}(A)$ in Fig. 5a, there exist critical tilt angles each for $P_{\mathrm{D}}\left(r_{\mathrm{d}}\right)$ and $P_{\mathrm{FA}}$, that maximize the said quantities. Since $\mathbb{P}(A)$ drops to zero beyond $\beta_{\mathrm{tilt}} \geq-\sqrt{\frac{A_{\mathrm{m}}}{12}} \beta_{3 \mathrm{~dB}}$ and regardless of the particular value of $\beta_{\text {tilt }}$, the LoS interference signal experiences a constant SLL of $-20 \mathrm{~dB}, P_{\mathrm{D}}\left(r_{\mathrm{d}}\right)$ and $P_{\mathrm{FA}}$ remains constant beyond the threshold beam tilt angles $\beta^{\prime \prime}{ }_{\text {tilt, }} P_{\mathrm{D}}$ and $\beta^{\prime \prime}{ }_{\text {tilt, } P_{\mathrm{FA}}}$. Since $\mathbb{P}(B)$ increases beyond zero for $\beta_{\mathrm{ub}} \geq 0^{\circ}$, the receive gain experienced by the LoS SOI and the respective $P_{\mathrm{D}}\left(r_{\mathrm{d}}\right)$ in Fig. 8b improve significantly for $\beta_{\mathrm{tilt}} \geq \beta_{\mathrm{d}}-\sqrt{\frac{A_{\mathrm{m}}}{12}} \beta_{3 \mathrm{~dB}}$.

2) Impact of $H P B W$ : Fig. 8a also shows that when $\mathbb{P}(B)=0$, as $\beta_{3 \mathrm{~dB}}$ increases, $P_{\mathrm{D}}\left(r_{\mathrm{d}}\right)$ and $P_{\mathrm{FA}}$ for all $\beta_{\text {tilt }}$ increase. This is due to the fact that as $\beta_{3 \mathrm{~dB}}$ increases, the power of both the NLoS drone signal and the NLoS interference signal increase for all $\beta_{\text {tilt }}$, increasing both $P_{\mathrm{D}}\left(r_{\mathrm{d}}\right)$ and $P_{\mathrm{FA}}$. Moreover, as $\beta_{3 \mathrm{~dB}}$ increases, the critical beam tilts $\beta_{\text {tilt, } \mathrm{P}_{\mathrm{D}}}^{\prime}$ and $\beta_{\text {tilt, } \mathrm{P}_{\mathrm{FA}}}^{\prime}$, move towards the lower bound of the $\beta_{\text {tilt }}$. This is because, the interference zone for a wider $\beta_{3 \mathrm{~dB}}$ sweeps through the high probability density interval of $R_{1, \mathrm{u}}$ at a faster rate with respect to the $\beta_{\text {tilt }}$, and achieves the maximum of $\mathbb{P}(A)$ sooner at a more negative tilt angle. Similarly, Fig. $8 \mathrm{~b}$ illustrates that when $\mathbb{P}(B) \geq 0$, as $\beta_{3 \mathrm{~dB}}$ increases from $8^{\circ}$ to $12^{\circ}$, the range of $\beta_{\text {tilt }}$ that allows for an improved $P_{\mathrm{D}}\left(r_{\mathrm{d}}\right)$ gets wider, due to the increase in the width of the interference zone.

3) Impact of $A 2 G$ Propagation: Contrary to the common intuition, Fig. 8a demonstrates that when $\mathbb{P}(B)=0, P_{\mathrm{D}}\left(r_{\mathrm{d}}\right)$ in an urban environment is higher than the $P_{\mathrm{D}}\left(r_{\mathrm{d}}\right)$ in a suburban environment, for all beam tilt angles. Due to the lack of alignment, the LoS component of the SOI experiences a very low receive gain, whereas the NLoS components experience 


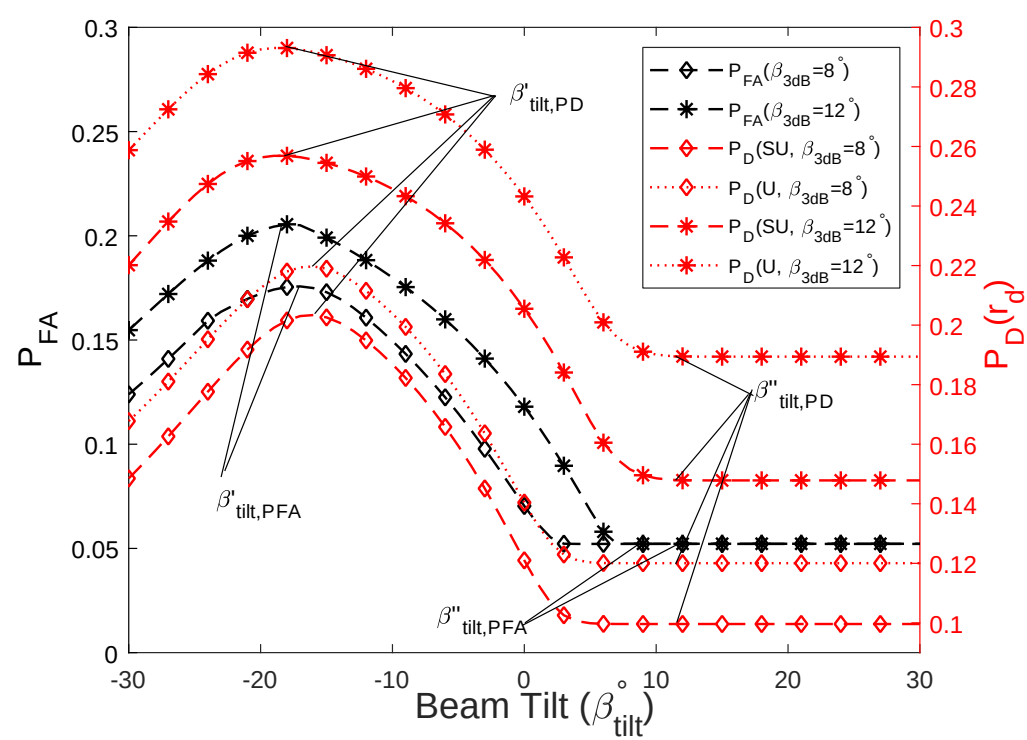

(a) Impact of HPBW ( $\left.\beta_{3 \mathrm{~dB}}\right)$ for $\beta_{\mathrm{d}}=85^{\circ}$ and $\mathbb{P}(B)=0$.

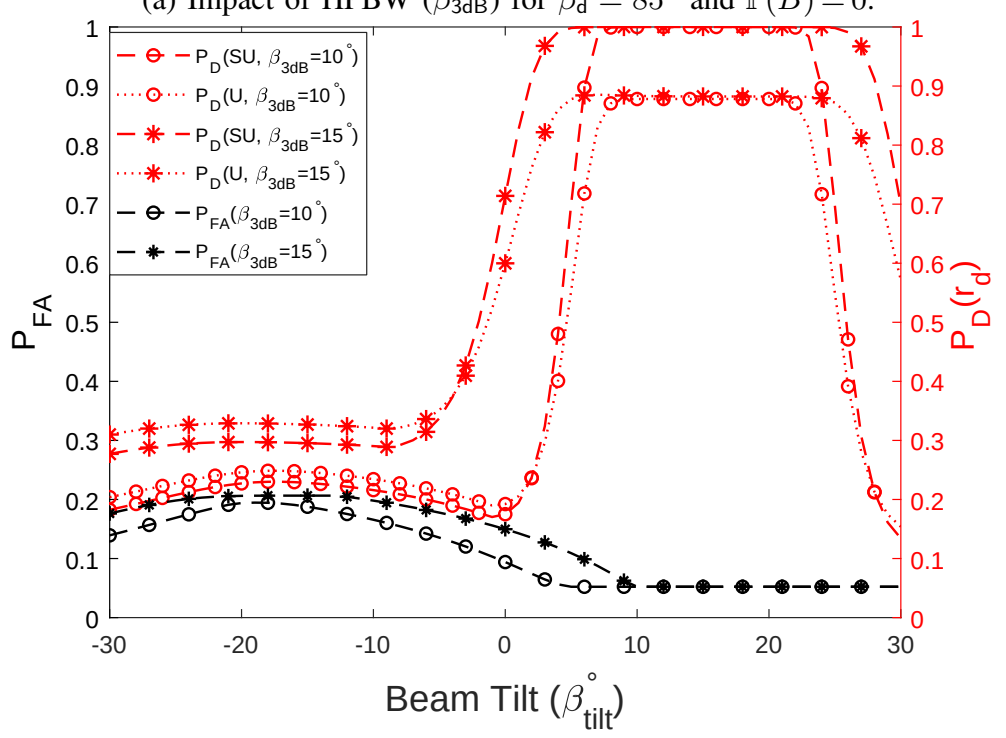

(b) Impact of HPBW ( $\beta_{3 \mathrm{~dB}}$ ) for $\beta_{\mathrm{d}}=15^{\circ}$ and $\mathbb{P}(B) \geq 0$.

Fig. 8: $P_{\mathrm{D}}\left(r_{\mathrm{d}}\right)$ and $P_{\mathrm{FA}}$ versus $\beta_{\text {tilt }}\left(\gamma_{\mathrm{I}}=4, \lambda=10^{-5} \mathrm{~m}^{-2}, h_{\mathrm{d}}=100 \mathrm{~m}\right.$, 'directional').

much higher receive gain. Since the probability of NLoS is higher in an urban environment as compared to a suburban environment, the average power of the drone signal and the respective $P_{\mathrm{D}}\left(r_{\mathrm{d}}\right)$ is higher in an urban environment. Fig. $8 \mathrm{~b}$ shows that the range of $\beta_{\text {tilt }}$ for which $\mathbb{P}(B)>0$, the $P_{\mathrm{D}}\left(r_{\mathrm{d}}\right)$ in the suburban environment becomes higher than the $P_{\mathrm{D}}\left(r_{\mathrm{d}}\right)$ in the urban environment.

\section{E. Sensors with Directional Antenna Patterns: Average De- tection Performance}

In this section we analyze the impact of the antenna radiation pattern $\left(\beta_{\text {tilt }}\right.$ and $\left.\beta_{3 \mathrm{~dB}}\right)$, the drone height $\left(h_{\mathrm{d}}\right)$, the network density $(\lambda)$, the propagation parameters ('SU' vs 'U'), and the various choice of the probe sensor on the detection coverage performance with directional antenna patterns. Please note that, as opposed to the conditional probability of detection, in Sec.VII-D, the coverage probability discussed in this section, is not dependent on the link distance between the drone and the sensor, but on the sensor density itself. However due to space limitation, we only include our analysis of the impact of $\beta_{\text {tilt }}, h_{\mathrm{d}}$ and $\lambda$ on $P_{\mathrm{D} \text {,avg. }}$. For the ease of analysis, we divide the associated interval of $\beta_{\text {tilt }}$ in 3 contiguous sub-intervals, namely: $I_{1}=\left[-30^{\circ}, \beta_{\mathrm{tilt}, \mathrm{P}_{\mathrm{FA}}}^{\prime}\right), I_{2}=\left[\beta_{\mathrm{tilt}, \mathrm{P}_{\mathrm{FA}}}^{\prime},-\sqrt{\frac{A_{\mathrm{m}}}{12}} \beta_{3 \mathrm{~dB}}\right)$ and $I_{3}=\left(-\sqrt{\frac{A_{\mathrm{m}}}{12}} \beta_{3 \mathrm{~dB}}, 30^{\circ}\right]$, where $\beta_{\mathrm{tilt}, \mathrm{P}_{\mathrm{FA}}}^{\prime}$ is the critical tilt angle that maximizes the $P_{\mathrm{FA}}$, as mentioned in Fig. 8a. In $I_{1}, \mathbb{P}(B)$ remains zero and $\mathbb{P}(A)$ increases monotonically with respect to $\beta_{\text {tilt. }}$ In $I_{2}, \mathbb{P}(A)$ starts to drop and $\mathbb{P}(B)$ starts to increase. In $I_{3}, \mathbb{P}(A)$ drops to its minimum and remains constant in the rest of the interval, whereas $\mathbb{P}(B)$ keeps increasing with the increasing tilt angle.

1) Impact of Node Density: As $\lambda$ varies, both UE and sensor density change. Any variation in the UE density impacts both the $P_{\mathrm{FA}}$ and the $P_{\mathrm{D}, \text { Avg }}$ by changing the receive gain and the path loss of the LoS interference signal, and the variance 


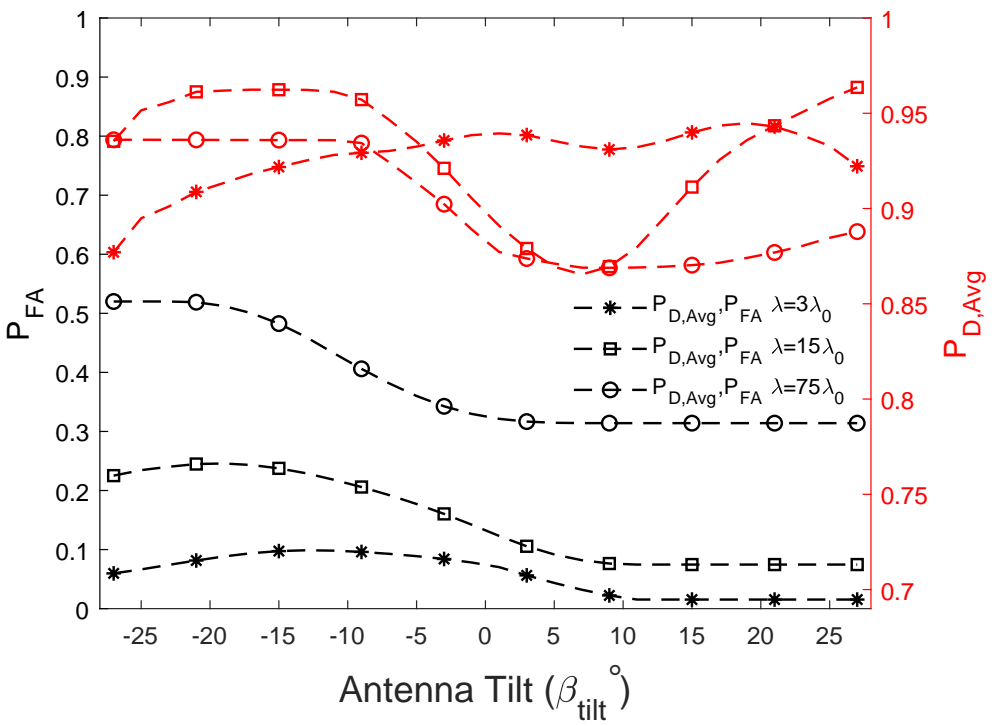

(a) Impact of node density $(\lambda)$ for ' $\mathrm{NN}-1$ '.

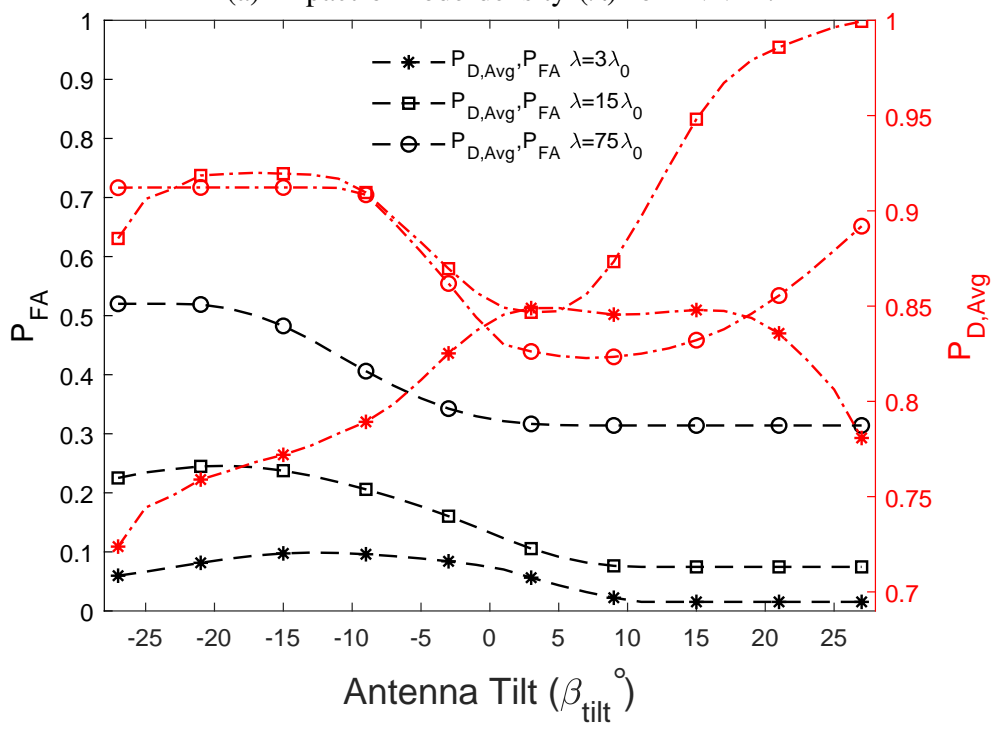

(b) Impact of node density $(\lambda)$ for 'NN-2'.

Fig. 9: $P_{\mathrm{D}, \text { avg }}$ for $h=135 \mathrm{~m}$, as a function of $\beta_{\text {tilt }}$ and $\lambda\left(\beta_{3 \mathrm{~dB}}=12^{\circ}, \gamma_{\mathrm{I}}=4\right.$, 'SU', '3GPP-like').

of the NLoS interference signal, whereas changes in the sensor density only impacts the $P_{\mathrm{D}, \text { Avg }}$ by changing the receive gain and the path loss experienced by the LoS SoI. However, the changes in the receive gain with respect to the increased $\lambda$ can be very different for different values of the sensor beam tilt.

For example, In Fig. 9a and Fig. 9b, we see that in the $I_{1}$ interval, $P_{\mathrm{FA}}$ and $P_{\mathrm{D}, \mathrm{Avg}}$ increase with $\lambda$ increasing from $3 \lambda_{0}$ to $75 \lambda_{0}$. This is because in $I_{1}$, an increasing $\lambda$ leads to higher $\mathbb{P}(A)$, higher receive gain, and lower path loss of the LoS interference signal. All of these factors together create an increasing pattern in the composite RSS and $P_{\mathrm{D}, \text { Avg }}$ with respect to the node density $\lambda$. However in the $I_{2}$ interval (where $\frac{d \mathbb{P}(A)}{d \beta_{\text {titt }}}<0$ and $\frac{d \mathbb{P}(B)}{d \beta_{\text {titt }}} \geq 0$ ) the $P_{\mathrm{D} \text {,avg }}$ can be decreasing or non-monotonic with respect to the $\lambda$. This is because when $\lambda$ increases, the path loss of the SOI and the interference signal decrease but the receive gain of the LoS SOI and LoS interference signal also decrease. Since in the $I_{2}$ interval we have: $\frac{d \mathbb{P}(A)}{d \beta_{\text {titl }}}<0$, we also have: $\frac{d \mathbb{P}(A)}{d \lambda}<0$, which leads to decreasing receive gain of the $\operatorname{LoS}$ interference signal with respect to $\lambda$. Similarly, due to having $\frac{d \mathbb{P}(B)}{d \beta_{\text {tilt }}} \geq 0$ in $I_{2}$, we also have: $\frac{d \mathbb{P}(B)}{d \lambda}<0$, which in turn causes the receive gain of the LoS SOI to decrease with an increasing $\lambda$. Since in $I_{3}$ we have: $\mathbb{P}(A)=0, \mathbb{P}(B)>0$ and $\frac{d \mathbb{P}(B)}{d \beta_{\text {titt }}}<0$, we also have: $\frac{d \mathbb{P}(B)}{d \lambda}>0$. Thus as $\lambda$ increases, the receive gain of LoS SOI also increases. This combined with the decreasing pathloss of the SOI and the interference signal causes the $P_{\mathrm{D} \text {,avg }}$ to be increasing with respect to the $\lambda$. Thus we realize that for a given $\beta_{\text {tilt }}$ there might exist a critical node density $\lambda_{\mathrm{c}}$ that maximizes $P_{\mathrm{D} \text {,avg }}$. As illustrated in Fig. 11a we see that, as the given $\beta_{\text {tilt }}$ becomes steeper, the value of the $\lambda_{c}$ also increases.

Comparing the $P_{\mathrm{D} \text {,avg }}$ and the $P_{\mathrm{FA}}$ for a particular node density (either $3 \lambda_{0}, 15 \lambda_{0}$ or $\left.75 \lambda_{0}\right)$, across the 3 intervals $\left(I_{1}\right.$, $I_{2}$ and $I_{3}$ ) we realize that the optimal detection coverage performance is attained at a certain critical tilt angle in $I_{3}$, where the RSS of the composite signal is maximized, but 


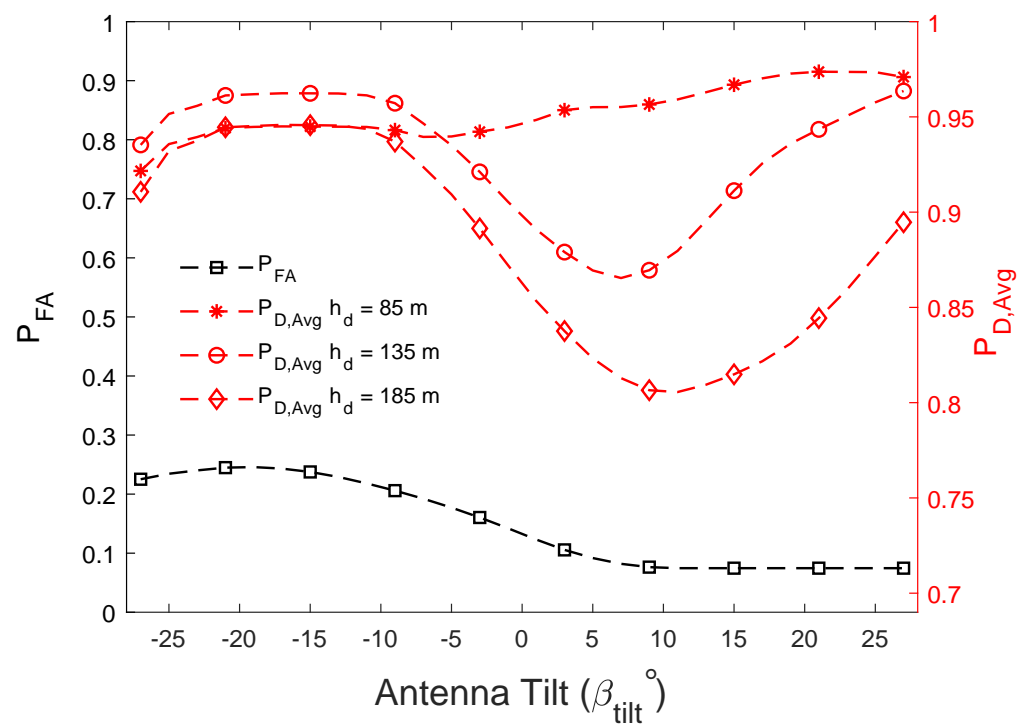

(a) Impact of drone altitude $\left(h_{\mathrm{d}}\right)$ for ' $\mathrm{NN}-1$ '.

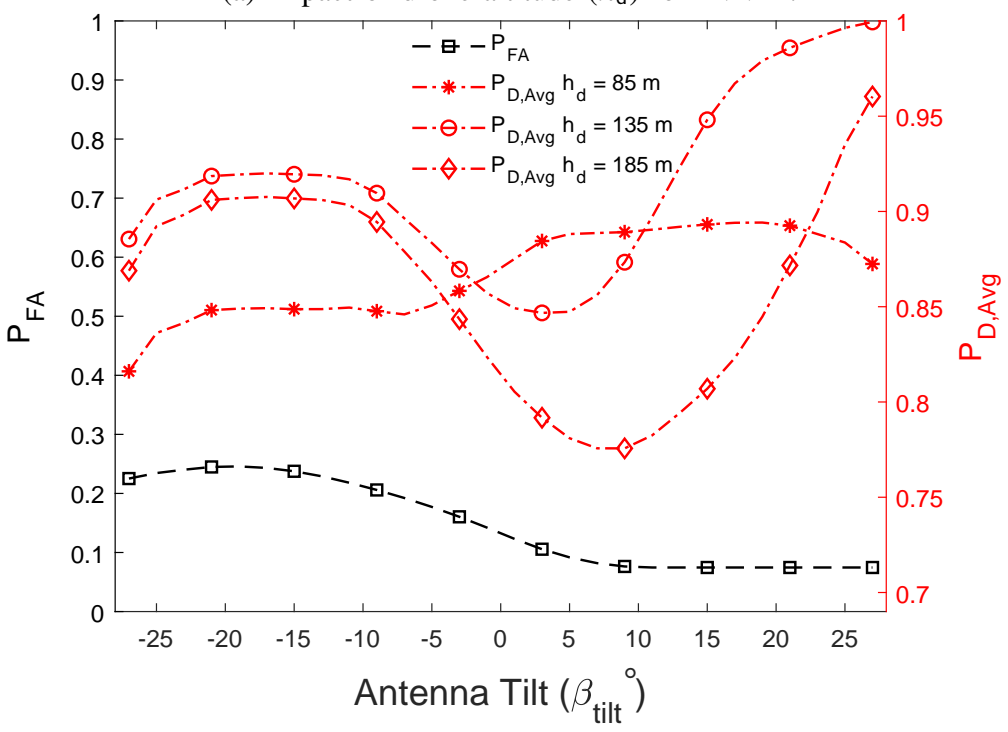

(b) Impact of drone altitude $\left(h_{\mathrm{d}}\right)$ for ' $\mathrm{NN}-2$ '.

Fig. 10: $P_{\mathrm{D} \text {,avg }}$ for $\lambda=1.5 \times 10^{-5} \mathrm{~m}^{-2}$, as a function of $\beta_{\text {tilt }}$ and drone altitude $h_{\mathrm{d}}\left(\beta_{3 \mathrm{~dB}}=12^{\circ}, \gamma_{\mathrm{I}}=4\right.$, 'SU', '3GPP-like').

the amplitude of the interference signal is minimized in a probabilistic sense (as $\mathbb{P}(A)$ becomes 0 in $I_{3}$ ). As the density increases the critical tilt angle moves towards the upper bound of the $\beta_{\text {tilt }}$ range.

2) Impact of Drone Altitude: Regardless of the sensor beam tilt, an increase in the altitude $h_{\mathrm{d}}$ causes a drop in the SOI amplitude due to the increased 3D link distance. It also causes the $P_{\text {LoS }}^{\mathrm{A}}$ in (3) to increase (due to the higher elevation angle, $\beta_{\mathrm{d}}$ ), which in turn increases the average SOI amplitude. Thus we see that in $I_{1}$, where $\mathbb{P}(B)=0$, the $P_{\mathrm{D} \text {,avg }}$ under suitable parameter configuration may show a non-monotonic pattern with respect to the altitude (as in Fig. 10a, and Fig. 10b, the $P_{\mathrm{D}, \text { avg }}$ for $h_{\mathrm{d}}=85 \mathrm{~m}$, and $h_{\mathrm{d}}=185 \mathrm{~m}$ are both smaller than the $P_{\mathrm{D}, \text { avg }}$ for $h_{\mathrm{d}}=135 \mathrm{~m}$, in the $I_{1}$ interval).

However, since in $I_{2}$ we have: $\mathbb{P}(B)>0$ and $\frac{d \mathbb{P}(B)}{d \beta_{\mathrm{titl}}}>0$, we also have $\frac{d \mathbb{P}(B)}{d h_{\mathrm{d}}}<0$, which causes the receive gain of the SOI to decrease with the increasing $h_{\mathrm{d}}$. This combined with the increased 3D link distance and path loss creates a decreasing pattern in the $P_{\mathrm{D} \text {,avg }}$ with respect to $h_{\mathrm{d}}$ in the $I_{2}$ interval. Finally, in the $I_{3}$ interval, where $\mathbb{P}(B)>0$ and $\frac{d \mathbb{P}(B)}{d \beta_{\mathrm{titl}}}<0$, we also have: $\frac{d \mathbb{P}(B)}{d h_{\mathrm{d}}}>0$, which means that the receive gain increases as $h_{\mathrm{d}}$ increases. Since the increase in the $h_{\mathrm{d}}$ also increases the pathloss, this results in a nonmonotonic relationship between the $P_{\mathrm{D} \text {,avg }}$ and the $h_{\mathrm{d}}$ in the $I_{3}$ interval. Thus we realize that for certain tilt angles, there exists a critical drone altitude $h_{\mathrm{c}}$, that maximizes $P_{\mathrm{D} \text {,avg }}$ by overcoming the increased pathloss with the increased receive gain. In Fig. 11b we illustrate the impact of the tilt angle on the critical altitude. We observe that as the given $\beta_{\text {tilt }}$ increases from $5^{\circ}$ to $25^{\circ}$ the value of $h_{\mathrm{c}}$ becomes larger.

Comparing the $P_{\mathrm{D} \text {,avg }}$ and the $P_{\mathrm{FA}}$ for a particular drone height (either $85 \mathrm{~m}, 135 \mathrm{~m}$ or $185 \mathrm{~m}$ ), across the 3 intervals $\left(I_{1}, I_{2}\right.$ and $\left.I_{3}\right)$ we realize that the optimal detection coverage performance is attained at a certain critical tilt angle in $I_{3}$, that maximizes the $P_{\mathrm{D} \text {,avg }}$ by maximizing $\mathbb{P}(B)$ and minimizes the 


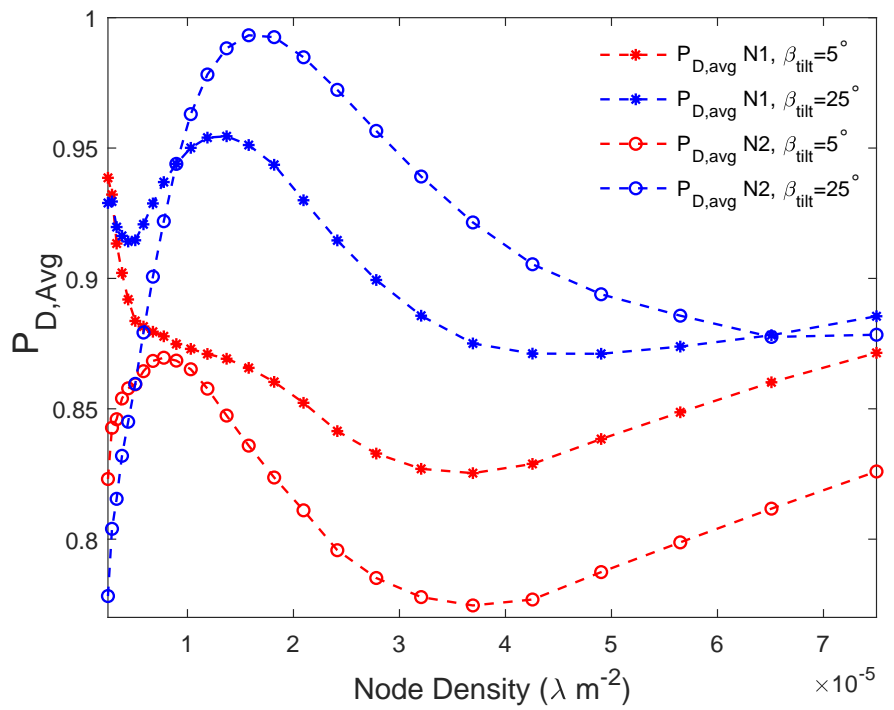

(a) Critical node density $\left(\lambda_{c}\right)$ for $\beta_{\text {tilt }} \in\left\{5^{\circ}, 25^{\circ}\right\}$.

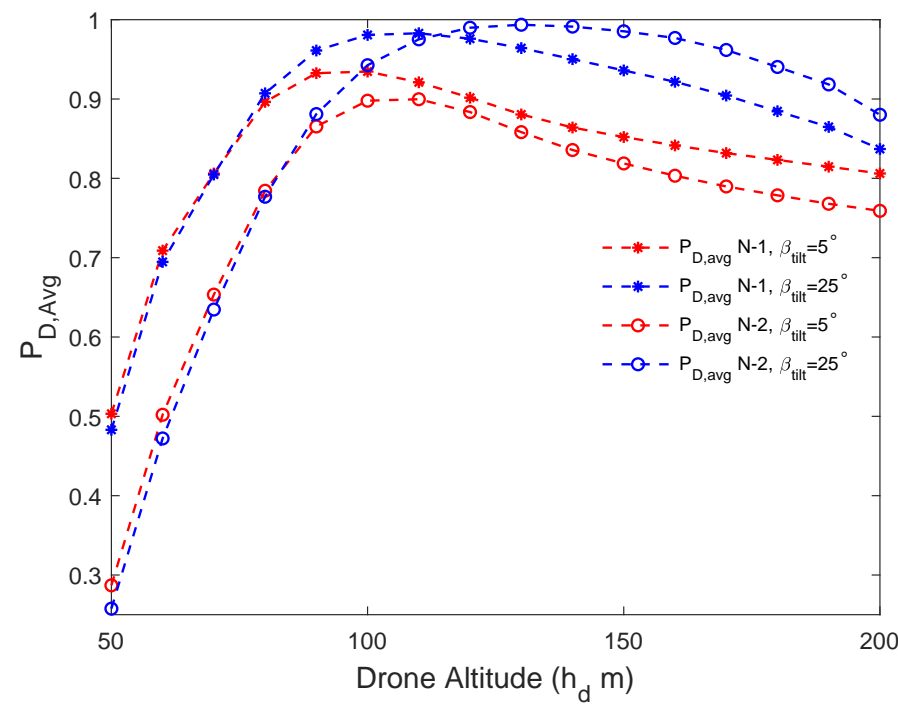

(b) Critical drone altitude $\left(h_{\mathrm{c}}\right)$ for $\beta_{\text {tilt }} \in\left\{5^{\circ}, 25^{\circ}\right\}$.

Fig. 11: Critical $\lambda$, and critical $h_{\mathrm{d}}$ of $P_{\mathrm{D}, \text { avg }}$ for 'NN-1', and 'NN-2' ( $\beta_{3 \mathrm{~dB}}=12^{\circ}, \gamma_{\mathrm{I}}=4$, 'SU', '3GPP-like').

$P_{\mathrm{FA}}$ by letting $\mathbb{P}(A)$ drop to zero. As the altitude increases the critical tilt angle moves towards the upper bound of the $\beta_{\text {tilt }}$ range.

3) Impact of Choosing the $n^{\text {th }}$ Nearest Sensor: The plots in Fig. 9a and Fig. 9a along with the plots in Fig. 10a and Fig. 10b, illustrate that when $\mathbb{P}(B ; 2)>\mathbb{P}(B ; 1)$ and the increased receive gain is higher than the increased pathloss, the $2^{\text {nd }}$ nearest sensor (denoted as ' $N-2$ ') yields a higher $P_{\mathrm{D} \text {,avg }}$, as compared to the the nearest sensor (denoted as ' $\mathrm{N}-1$ '). Thus both in Fig. 9a and Fig. 9b, and Fig. 10a and Fig. 10b, at very steep positive angles, $P_{\mathrm{D}, \mathrm{Avg}}$ yielded by ' $\mathrm{N}-2$ ' is higher than that yielded by ' $\mathrm{N}-1$ '. While for very low node density $\left(\lambda=3 \lambda_{0}\right)$ and very low drone altitude $\left(h_{\mathrm{d}}=135 \mathrm{~m}\right)$ ' $\mathrm{N}-1$ ' is preferred at all $\beta_{\text {tilt }}$, as $\lambda$ and $h_{\mathrm{d}}$ increase, the threshold beam tilt beyond which the ' $\mathrm{N}-2$ ' is preferred over the ' $\mathrm{N}-1$ ', becomes steeper.

\section{CONCLUSION}

In this work we introduce an analytical framework to study the joint impact of the sensor and the UE densities, A2G channel characteristics, and 3D antenna patterns, on RF-based detection of drones by a network of ground RF sensors. In particular, we derive analytical expressions for the probabilities of detection and false alarm at each individual sensor, as well as the coverage probability of detection for the entire sensor network, considering both isotropic and directional antenna patterns. Our analysis reveals that there exists a critical sensor density and critical beam tilt that optimize the detection coverage. Contrary to the common intuition, we discover the benefits of using far-flung sensors as opposed to the nearest sensor, and thus are able to select the best probe sensor, among all the sensors in the sensing network, that optimizes the detection coverage probability. Our findings can help in using existing/future terrestrial RF networks for detecting drones, which in turn can improve the safety of unmanned aerial traffic and urban air mobility in the future.

\section{APPENDIX}

\section{A. Baseband Signal Representation}

The I-Q components of $\boldsymbol{Z}=Z_{\mathrm{I}}+j Z_{\mathrm{Q}}$ are obtained by projecting the respective random process $Z(t)$ onto a set of orthonormal basis functions: $\left\{f_{\mathrm{I}}(t)=\sqrt{2} g_{\mathrm{u}}(t) \cos \left(2 \pi f_{\mathrm{c}} t\right)\right.$, $\left.f_{\mathrm{Q}}(t)=-\sqrt{2} g_{\mathrm{u}}(t) \sin \left(2 \pi f_{\mathrm{c}} t\right)\right\} \quad$ [32]. We show the I-Q decomposition of $\boldsymbol{Z}$ for the LoS case, in (??). Using $g_{\mathrm{u}}(t)=g_{\mathrm{d}}(t)=u(t)$ as in (19) and $\tau_{\mathrm{d}} \sim \mathcal{U}[0, T]$ in Section V, we get $\rho_{\mathrm{d}}=\int_{0}^{T} u(t) u\left(t-\tau_{\mathrm{d}}\right) \mathrm{d} t=\int_{\tau_{\mathrm{d}}}^{T} \frac{1}{T} \mathrm{~d} t=1-\frac{\tau_{\mathrm{d}}}{T}$ and thus we get: $\rho_{\mathrm{d}} \sim \mathcal{U}[0,1]$. Similarly we have $\rho_{i, \mathrm{u}} \sim \mathcal{U}[0,1]$, for $1 \leq i<\infty$. Following similar algebraic simplifications we can show that

$$
\begin{aligned}
& Z_{\mathrm{Q}}=\sum_{m=1}^{M_{\mathrm{d}}} \frac{k \rho_{\mathrm{d}} \alpha_{m} \sqrt{P_{\mathrm{d}} G\left(\beta_{m}\right)} \sin \left(\phi_{m}+\theta_{\mathrm{d}}\right)}{d_{\mathrm{LoS}} \sqrt{\eta^{\mathrm{NLoS}}}} \\
& +\frac{k \rho_{\mathrm{d}} \sqrt{P_{\mathrm{d}} G\left(\beta_{\mathrm{d}}\right)} \sin \left(\theta_{\mathrm{d}}\right)}{d_{\mathrm{LoS}} \sqrt{\eta^{\text {LoS }}}}, \\
& Y_{\mathrm{I}}=\sum_{i=1}^{\infty} \sum_{m=1}^{M_{\mathrm{u}}} \frac{k^{\frac{\gamma_{\mathrm{I}}}{2}} \alpha_{m, i} \rho_{i, \mathrm{u}}}{R_{i, \mathrm{u}}{ }^{\frac{\gamma_{\mathrm{I}}}{2}}} \sqrt{P_{\mathrm{u}} G\left(\beta_{m, i}\right)} \cos \left(\theta_{i, \mathrm{u}}+\phi_{m, i}\right) \\
& +\frac{k^{\frac{\gamma_{\mathrm{L}}}{2}} \rho_{1, \mathrm{u}} \sqrt{P_{\mathrm{u}} G\left(\beta_{1, \mathrm{u}}\right)} \cos \left(\theta_{1, \mathrm{u}}\right)}{\left(R_{1, \mathrm{u}}\right)^{\frac{\gamma_{\mathrm{L}}}{2}}} \\
& Y_{\mathrm{Q}}=\sum_{i=1}^{\infty} \sum_{m=1}^{M_{\mathrm{u}}} \frac{k^{\frac{\gamma_{1}}{2}} \alpha_{m, i} \rho_{i, \mathrm{u}}}{R_{i, \mathrm{u}}{ }^{\frac{\gamma_{1}}{2}}} \sqrt{P_{\mathrm{u}} G\left(\beta_{m, i}\right)} \sin \left(\theta_{i, \mathrm{u}}+\phi_{m, i}\right) \\
& +\frac{k^{\frac{\gamma_{\mathrm{L}}}{2}} \rho_{1, \mathrm{u}} \sqrt{P_{\mathrm{u}} G\left(\beta_{1, \mathrm{u}}\right)} \sin \left(\theta_{1, \mathrm{u}}\right)}{\left(R_{1, \mathrm{u}}\right)^{\frac{\gamma_{\mathrm{L}}}{2}}} \text {. }
\end{aligned}
$$

\section{B. Distribution of Aggregate Interference Amplitude}

We will consider two cases separately; first the NLoS setting and then the LoS setting. 


$$
\begin{aligned}
& Z_{\mathrm{I}}=\sum_{m=1}^{M} \int_{0}^{T}\left[\frac{k \alpha_{m}}{d_{\mathrm{LoS}}} \frac{\sqrt{2 P_{\mathrm{d}} G\left(\beta_{m}\right)}}{\sqrt{\eta^{\text {NLOS }}}} g_{\mathrm{d}}\left(t-\tau_{\mathrm{d}}\right) \cos \left(2 \pi f_{\mathrm{c}} t+\theta_{\mathrm{d}}+\phi_{m}\right) \times \sqrt{2} g_{\mathrm{u}}(t) \cos \left(2 \pi f_{\mathrm{c}} t\right)\right] \mathrm{d} t \\
& +\int_{0}^{T}\left[\frac{k}{d_{\mathrm{LoS}}} \frac{\sqrt{2 P_{\mathrm{d}} G\left(\beta_{\mathrm{d}}\right)}}{\sqrt{\eta^{\operatorname{LoS}}}} g_{\mathrm{d}}\left(t-\tau_{\mathrm{d}}\right) \cos \left(2 \pi f_{\mathrm{c}} t+\theta_{\mathrm{d}}\right) \times \sqrt{2} g_{\mathrm{u}}(t) \cos \left(2 \pi f_{\mathrm{c}} t\right)\right] \mathrm{d} t \\
& =\sum_{m=1}^{M} \frac{k \alpha_{m} \cos \left(\theta_{\mathrm{d}}+\phi_{m}\right) \sqrt{P_{\mathrm{d}} G\left(\beta_{m}\right)}}{d_{\mathrm{LoS}} \sqrt{\eta_{n \mathcal{N}}}} \underbrace{\int_{0}^{T} g_{\mathrm{d}}\left(t-\tau_{\mathrm{d}}\right) g_{\mathrm{u}}(t) \mathrm{d} t}_{=\rho_{\mathrm{d}}}+\frac{k \cos \left(\theta_{\mathrm{d}}\right) \sqrt{P_{\mathrm{d}} G\left(\beta_{\mathrm{d}}\right)}}{d_{\operatorname{Los}} \sqrt{\eta^{\operatorname{LoS}}}} \underbrace{\int_{0}^{T} g_{\mathrm{d}}\left(t-\tau_{\mathrm{d}}\right) g_{\mathrm{u}}(t) \mathrm{d} t}_{=\rho_{\mathrm{d}}} .
\end{aligned}
$$

Case $1\left(\mathrm{~s}_{\mathrm{T}}=\mathrm{NLoS}\right)$ : Using complex random variables $\boldsymbol{U}_{i}$, $\boldsymbol{X}_{i}, \boldsymbol{B}_{m, i}$, we rewrite (20) as:

$$
\boldsymbol{Y}=\sum_{i=1}^{\infty} \frac{\boldsymbol{U}_{i}}{\left(R_{i, \mathrm{u}}\right)^{\frac{\gamma_{1}}{2}}}, \quad \boldsymbol{U}_{i}=U_{i, \mathrm{I}}+j U_{i, \mathrm{Q}}
$$

where $U_{i, \mathrm{n}}=\rho_{i, \mathrm{u}} X_{i, \mathrm{n}}=\rho_{i, \mathrm{u}} \sum_{m=1}^{M_{\mathrm{d}}} B_{m, i, \mathrm{n}}$, and ,

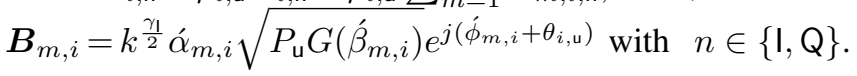

Distribution of $U_{i, \mathrm{n}}$ : Using moment properties of the respective random variables we can show that for all $i$ and $m, \mathbb{E}\left[B_{m, i, \mathrm{Q}}\right]=0$, and

$$
\begin{aligned}
\mathbb{E}\left[B_{m, i, \mathrm{Q}}^{2}\right] & =k^{\frac{\gamma_{1}}{2} 2} P_{\mathrm{u}} \mathbb{E}\left[\alpha^{2}{ }_{m, i}\right] \mathbb{E}\left[G\left(\dot{\beta}_{m}\right)\right] \\
& =k^{\frac{\gamma_{1}}{2} 2} P_{\mathrm{u}} \int_{-\frac{\pi}{2}}^{0} G\left(\dot{\beta}_{m}\right) f_{\dot{\beta}_{m}}\left(\dot{\beta}_{m}\right) \mathrm{d} \dot{\beta} \\
& =k^{\frac{\gamma_{1}}{2}} P_{\mathrm{u}} \frac{h\left(\beta_{3 \mathrm{~dB}}\right)}{2}
\end{aligned}
$$

where the expression for $h\left(\beta_{3 d B}\right)$ is the same as in (25), which is not dependent on either $m$ or $i$. Since $\left\{\dot{\alpha}_{m, i}\right\},\left\{\hat{\beta}_{m, i}\right\}$, and $\left\{\dot{\phi}_{m, i}\right\}$ are sequences of random variables that are i.i.d in both $m$ and $i,\left\{B_{m, i, \mathrm{n}}\right\}$ is also a sequence of random variables, i.i.d in both $m$ and $i$, for both $n \in\{\mathrm{I}, \mathrm{Q}\}$. Now using CLT [31] we see that $X_{i, \mathrm{Q}}=\sum_{m=1}^{M_{\mathrm{u}}} B_{m, i, \mathrm{Q}} \stackrel{d}{\rightarrow} \mathcal{N}\left(0, \sigma^{2}\right)$, as $M_{\mathrm{u}} \rightarrow$ $\infty$ where $\sigma^{2}=\mathbb{E}\left[B_{m, i, \mathrm{Q}}^{2}\right]$ as in (43). Similarly we can show $X_{i, \mathrm{I}}=\sum_{m=1}^{M_{u}} B_{m, i, \mathrm{I}} \stackrel{d}{\rightarrow} \mathcal{N}\left(0, \sigma^{2}\right)$.

Since $\left\{B_{m, i, 1}\right\}$ and $\left\{B_{m, i, \mathrm{Q}}\right\}$ are two sequences of i.i.d random variables in $i$, we conclude that $\left\{X_{i, \mathrm{n}}\right\}$ is a sequence of i.i.d Gaussian random variables for with $X_{i, \mathrm{n}} \sim \mathcal{N}\left(0, \sigma^{2}\right)$ for all $i$, and both $n \in\{\mathrm{I}, \mathrm{Q}\}$. Similarly we realize that $U_{i, \mathrm{n}}$ is also a sequence of random variables that are i.i.d in $i$, since $U_{i, \mathrm{n}}=\rho_{i, \mathrm{u}} X_{i, \mathrm{n}}$, and $\left\{\rho_{i, \mathrm{u}}\right\}$ and $\left\{X_{i, \mathrm{n}}\right\}$ are also sequences of random variables, i.i.d in $i$.

Distribution of $Y_{\mathrm{n}}$ : Since $\boldsymbol{U}_{\boldsymbol{i}}$ is a series of i.i.d random variables and $\left[R_{i, \mathrm{u}}\right]_{i=1}^{\infty}$ is defined with respect to a PPP, $Y_{\mathrm{I}}$ and $Y_{\mathrm{Q}}$ become jointly Stable random variables [33], such that $Y_{\mathrm{n}} \sim \mathcal{S}_{c}\left(\alpha_{\mathrm{Y}}, \beta_{\mathrm{Y}}, \gamma_{\mathrm{Y}}\right)$, with $\alpha_{\mathrm{Y}}=\frac{4}{\gamma_{1}}, \beta_{\mathrm{Y}}=0$, and $\gamma_{\mathrm{Y}}=$ $\pi \lambda k_{\mathrm{u}} \mathrm{C}_{\frac{4}{\gamma_{1}}}^{-1} E\left\{\left|U_{i, \mathrm{n}}\right|^{\frac{4}{\gamma_{1}}}\right\}$, where

$$
C_{\mathrm{x}}=\left\{\begin{array}{ll}
\frac{2}{\pi}, & \text { for } x=1 \\
\frac{1}{\pi} \sin \left(\frac{\pi}{2 x}\right) \Gamma(x), & \text { otherwise }
\end{array} .\right.
$$

Now using the moment properties of the Gaussian $\left(Q_{\mathrm{n}}\right)$ and the Uniform $\left(\rho_{i, \mathrm{u}}\right)$ random variables, we obtain for both $n \in$ $\{I, Q\}$ :

$$
E\left\{\left|U_{i, \mathrm{n}}\right|^{\frac{4}{\gamma_{1}}}\right\}=\frac{1}{\pi^{\gamma_{1}}} k_{l_{1}}^{\frac{4}{\gamma_{1}}} P_{\mathrm{u}^{\frac{2}{\gamma_{1}}}}^{\frac{2}{\gamma_{\mathrm{I}}+2}} \Gamma\left(\frac{1}{2}+\frac{4}{\gamma_{\mathrm{l}}}\right) h\left(\beta_{3 \mathrm{~dB}}\right) .
$$

Decomposition of Stable Random Variables: Next, using the decomposition property of Stable random variables [33] we get $Y=\sqrt{V} \boldsymbol{G}$ such that $\boldsymbol{G}=G_{1}+j G_{\mathrm{Q}}$ is a complex Gaussian random variable such that for both $n \in\{I, Q\}, G_{\mathrm{n}} \sim \mathcal{N}_{c}\left(0,2 \gamma_{\mathrm{G}}\right)$, where $\gamma_{\mathrm{G}}=2\left(\gamma_{\mathrm{Y}}\right)^{\frac{\gamma_{1}}{2}}$, and $V \sim$ $\mathcal{S}\left(\alpha_{\mathrm{V}}, \beta_{\mathrm{V}}, \gamma_{\mathrm{V}}\right)$, with $\alpha_{\mathrm{V}}=\frac{2}{\gamma_{1}}, \beta_{\mathrm{V}}=1, \gamma_{\mathrm{V}}=\cos \left(\frac{\pi}{\gamma_{1}}\right)$, and $V$ and $\boldsymbol{G}$ are independent random variables [33]. Therefore, $Y_{\mid} \mid V$ and $Y_{\mathrm{Q}} \mid V$ are i.i.d random variables with $Y_{\mathrm{n}} \mid V \sim \mathcal{N}\left(0, \sigma_{\mathrm{y}}{ }^{2}\right)$, where $\sigma_{\mathrm{y}}{ }^{2}=V \gamma_{\mathrm{G}}=2 V\left(\gamma_{\mathrm{Y}}\right)^{\frac{\gamma_{1}}{2}}$ as in (24).

Case $2\left(\mathrm{~s}_{\mathrm{T}}=\mathrm{LoS}\right)$ : Using the random variables $U_{i, \mathrm{I}}$ and $U_{i, \mathrm{Q}}$ as in Case $1\left(\mathrm{~s}_{\mathrm{T}}=\mathrm{NLoS}\right)$, we rewrite (20) as in (40). Using $\left(\sum_{i=1}^{\infty} \frac{U_{i, \mathrm{n}}}{\left(R_{i, \mathrm{u}}\right)^{\frac{\gamma 1}{2}}}\right) \mid V \sim \mathcal{N}\left(0, \sigma_{\mathrm{y}}{ }^{2}\right)$, from Case 1 ( $\mathrm{s}_{\mathrm{T}}=$ NLoS), the distribution of $Y_{\mathrm{I}}$ and $Y_{\mathrm{Q}}$ in (40), are given as in (41) and (42), respectively.

\section{Distribution of SOI}

Let's define

$$
B_{m}=\frac{k \rho_{\mathrm{d}} \alpha_{m} \sqrt{P_{\mathrm{d}} G\left(\beta_{m}\right)} \sin \left(\phi_{m}+\theta_{\mathrm{d}}\right)}{d_{\mathrm{LoS}} \sqrt{\eta^{\mathrm{NLOS}}}}
$$

where $\mathbb{E}\left[B_{m} \mid R_{\mathrm{d}}, \rho_{\mathrm{d}}, \theta_{\mathrm{d}}\right]=0$. Since $\left\{\alpha_{m}\right\},\left\{\phi_{m}\right\}$ and $\left\{\beta_{m}\right\}$ are sequences of i.i.d random variables, $\left\{B_{m} \mid R_{\mathrm{d}}, \rho_{\mathrm{d}}, \theta_{\mathrm{d}}\right\}$ is also a sequence of i.i.d random variables. Thus for a large value of $M_{\mathrm{d}}$ [31], by the application of the Central Limit Theorem (CLT) we have the following cases.

Case $1\left(s_{A}=N L O S\right)$ :

$$
\begin{aligned}
& Z_{\mathrm{n}} \sim \mathcal{N}\left(0, \sigma_{\mathrm{z}}^{2}\right) \\
& \sigma_{\mathrm{z}}^{2}=\mathbb{E}\left[B_{m}^{2} \mid R_{\mathrm{d}}, \rho_{\mathrm{d}}, \theta_{\mathrm{d}}\right], \forall \mathrm{n} \in\{\mathrm{I}, \mathrm{Q}\},
\end{aligned}
$$

where $\mathbb{E}\left[B_{m}^{2} \mid R_{\mathrm{d}}, \rho_{\mathrm{d}}, \theta_{\mathrm{d}}\right]$ is computed as in (46). 


$$
\begin{aligned}
Y_{\mathrm{I}} & =\sum_{i=1}^{\infty} \frac{U_{i, \mathrm{I}}}{\left(R_{i, \mathrm{u}}\right)^{\frac{\gamma_{1}}{2}}}+\rho_{1, \mathrm{u}}\left(\frac{k}{R_{1, \mathrm{u}}}\right)^{\frac{\gamma_{\mathrm{L}}}{2}} \sqrt{P_{\mathrm{u}} G\left(\beta_{1, \mathrm{u}}\right)} \cos \left(\theta_{1, \mathrm{u}}\right), \\
Y_{\mathrm{Q}} & =\sum_{i=1}^{\infty} \frac{U_{i, \mathrm{Q}}}{\left(R_{i, \mathrm{u}}\right)^{\frac{\gamma_{\mathrm{l}}}{2}}}+\rho_{1, \mathrm{u}}\left(\frac{k}{R_{1, \mathrm{u}}}\right)^{\frac{\gamma_{\mathrm{L}}}{2}} \sqrt{P_{\mathrm{u}} G\left(\beta_{1, \mathrm{u}}\right)} \sin \left(\theta_{1, \mathrm{u}}\right) .
\end{aligned}
$$

$$
\begin{gathered}
Y_{\mathrm{I}} \mid R_{1, \mathrm{u}}, \rho_{1, \mathrm{u}}, \theta_{1, \mathrm{u}}, V \sim \mathcal{N}\left(\mu_{\mathrm{I}, \mathrm{Y}}, \sigma_{\mathrm{Y}}^{2}\right), \quad \text { with } \mu_{\mathrm{I}, \mathrm{Y}}=\frac{k \rho_{1, \mathrm{u}} \sqrt{P_{\mathrm{u}} G\left(\beta_{1, \mathrm{u}}\right)} \cos \left(\theta_{1, \mathrm{u}}\right)}{R_{1, \mathrm{u}}^{\gamma /}} \\
Y_{\mathrm{Q}} \mid R_{1, \mathrm{u}}, \rho_{1, \mathrm{u}}, \theta_{1, \mathrm{u}}, V \sim \mathcal{N}\left(\mu_{\mathrm{Q}, \mathrm{Y}}, \sigma_{\mathrm{Y}}^{2}\right), \quad \text { with } \mu_{\mathrm{Q}, \mathrm{Y}}=\frac{k \rho_{1, \mathrm{u}} \sqrt{P_{\mathrm{u}} G\left(\beta_{1, \mathrm{u}}\right)} \sin \left(\theta_{1, \mathrm{u}}\right)}{R_{1, \mathrm{u}}^{\gamma / \mathrm{L}}}
\end{gathered}
$$

$$
\begin{aligned}
& \mathbb{E}\left[B_{m}^{2} \mid R_{\mathrm{d}}, \rho_{\mathrm{d}}, \theta_{\mathrm{d}}\right]=\frac{k^{2} P_{\mathrm{d}} \rho_{\mathrm{d}}^{2}}{d_{\mathrm{LoS}}^{2} \eta^{\mathrm{NLOS}}} \mathbb{E}\left[\alpha_{m}^{2}\right] \mathbb{E}\left[\sin ^{2}\left(\phi_{m}+\theta_{\mathrm{d}}\right)\right] \mathbb{E}\left[G\left(\beta_{m}\right)\right] \\
& =\frac{k^{2} P_{\mathrm{d}} \rho_{\mathrm{d}}^{2}}{2 d_{\text {Los }}^{2} \eta^{\mathrm{NLoS}}} \mathbb{E}\left[G\left(\beta_{m}\right)\right]=\frac{k^{2} P_{\mathrm{d}} \rho_{\mathrm{d}}^{2}}{2 d_{\text {LoS }}^{2} \eta^{\mathrm{NLoS}}} \int_{-\frac{\pi}{2}}^{\frac{\pi}{2}} G\left(\beta_{m}\right) f_{\beta_{m}}\left(\beta_{m}\right) \mathrm{d} \beta_{m} \\
& =\frac{k^{2} P_{\mathrm{d}} \rho_{\mathrm{d}}^{2}}{2 d_{\text {LoS }}^{2} \eta^{\mathrm{NLoS}}}\left(\frac{e^{0.23 G_{\mathrm{P}, \max }}}{\pi} \int_{-\frac{\pi}{2}}^{\frac{\pi}{2}} \exp \left(\frac{\beta_{m}-\beta_{\text {tilt }}}{\beta_{3 \mathrm{~dB}}}\right)^{2} \mathrm{~d} \beta_{m}+\left(1-\frac{2 \beta_{3 \mathrm{~dB}}}{\pi} \sqrt{\frac{A_{\mathrm{m}}}{12}}\right) e^{\left(0.23\left(G_{\mathrm{P}, \max }-A_{\mathrm{m}}\right)\right)}\right) .
\end{aligned}
$$

Case $2\left(\mathrm{~s}_{\mathrm{A}}=\mathrm{LoS}\right)$ :

$$
\begin{aligned}
Z_{\mathrm{Q}} \mid R_{\mathrm{d}}, \rho_{\mathrm{d}}, \theta_{\mathrm{d}} & =\frac{k \rho_{\mathrm{d}} \sqrt{P_{\mathrm{d}} G\left(\beta_{\mathrm{d}}\right)} \sin \left(\theta_{\mathrm{d}}\right)}{d_{\mathrm{LoS}} \sqrt{\eta^{\operatorname{LoS}}}} \\
& +\sum_{m=1}^{M_{\mathrm{d}}} B_{m} \mid R_{\mathrm{d}}, \rho_{\mathrm{d}}, \theta_{\mathrm{d}} \stackrel{d}{\rightarrow} \mathcal{N}\left(\mu_{\mathrm{Q}, \mathrm{Z}}, \sigma_{\mathrm{z}}^{2}\right) \\
Z_{\mathrm{I}} \mid R_{\mathrm{d}}, \rho_{\mathrm{d}}, \theta_{\mathrm{d}} & =\frac{k \rho_{\mathrm{d}} \sqrt{P_{\mathrm{d}} G\left(\beta_{\mathrm{d}}\right)} \cos \left(\theta_{\mathrm{d}}\right)}{d_{\mathrm{LoS}} \sqrt{\eta^{\operatorname{LoS}}}} \\
& +\sum_{m=1}^{M_{\mathrm{d}}} B_{m} \mid R_{\mathrm{d}}, \rho_{\mathrm{d}}, \theta_{\mathrm{d}} \stackrel{d}{\rightarrow} \mathcal{N}\left(\mu_{\mathrm{l}, \mathrm{z}}, \sigma_{\mathrm{z}}{ }^{2}\right),
\end{aligned}
$$

where

$$
\begin{aligned}
\mu_{\mathrm{Q}, \mathrm{Z}} & =\frac{k \rho_{\mathrm{d}} \sqrt{P_{\mathrm{d}} G\left(\beta_{\mathrm{d}}\right)} \sin \left(\theta_{\mathrm{d}}\right)}{d_{\mathrm{LoS}} \sqrt{\eta^{\mathrm{LoS}}}}, \\
\mu_{\mathrm{I}, \mathrm{Z}} & =\frac{k \rho_{\mathrm{d}} \sqrt{P_{\mathrm{d}} G\left(\beta_{\mathrm{d}}\right)} \cos \left(\theta_{\mathrm{d}}\right)}{d_{\mathrm{LoS}} \sqrt{\eta^{\operatorname{LoS}}}} .
\end{aligned}
$$

\section{Cumulative CDFs of RSS with Null Distribution}

We first derive the conditional distributions under the null and the alternative hypotheses

$$
\bar{F}_{R_{\mathrm{n}} \mid V, R_{1, \mathrm{u}}, \mathrm{S}_{\mathrm{T}}}\left(\gamma_{\mathrm{th}} \mid v, r_{1, \mathrm{u}}, \mathrm{ST} ; \mathcal{H}_{0}\right),
$$

and

$$
\bar{F}_{R_{\mathrm{n}} \mid V, R_{1, \mathrm{u}}, R_{\mathrm{d}}, \mathrm{S}_{\mathrm{T}}, \mathrm{s}_{\mathrm{A}}}\left(\gamma_{\mathrm{th}} \mid v, r_{1, \mathrm{u}}, r_{\mathrm{d}}, \mathrm{S}_{\mathrm{T}}, \mathrm{s}_{\mathrm{A}} ; \mathcal{H}_{1}\right),
$$

respectively.

Case $1\left(\mathrm{~s}_{\mathrm{T}}=\mathrm{NLOS}\right)$ : For this scenario, we have $\mathcal{H}_{0}$ : $R_{\mathrm{l}}, R_{\mathrm{Q}} \stackrel{\mid V}{\sim} \mathcal{N}\left(0, \sigma_{0}{ }^{2}\right)$. We now define a random variable:

$$
X_{0}=\frac{R_{\mathrm{s}}}{\sigma_{0}{ }^{2}}=\frac{R_{\mathrm{I}}{ }^{2}}{\sigma_{0}{ }^{2}}+\frac{R_{\mathrm{Q}}{ }^{2}}{\sigma_{0}{ }^{2}},
$$

where, $X_{0}$ conditioned on $V$, is distributed as a Chi-Squared random variable of degree 2: $X_{0} \stackrel{\mid V}{\sim} \chi_{2}^{2}$. Thus we obtain the CCDF of the RSS as:

$$
\begin{aligned}
\bar{F}_{R_{\mathrm{s}} \mid V}\left(r_{\mathrm{s}} ; \mathcal{H}_{0}\right) & =P\left(R_{\mathrm{s}} \geq r_{\mathrm{s}}\right) \\
& =P\left(X_{0} \geq \frac{r_{\mathrm{s}}}{\sigma_{0}^{2}}\right)=\exp \left(-\frac{r_{\mathrm{s}}}{2 \sigma_{0}^{2}}\right) .
\end{aligned}
$$

Case $2\left(\mathrm{~s}_{\mathrm{T}}=\mathrm{LoS}\right)$ : After scaling $R_{\mathrm{I}}$ and $R_{\mathrm{Q}}$ by $\sigma_{0}{ }^{2}$, we get $X_{0}=\frac{R_{\mathrm{S}}}{\sigma_{0}{ }^{2}}=\frac{R_{\mathrm{l}}{ }^{2}}{\sigma_{0}{ }^{2}}+\frac{R_{\mathrm{Q}}{ }^{2}}{\sigma_{0}{ }^{2}}$, and

$$
\begin{aligned}
\mathcal{H}_{0}: & \frac{R_{\mathrm{I}}}{\sigma_{1}} \stackrel{V, R_{1, \mathrm{u}}, \rho_{1, \mathrm{u}}}{\sim} \mathcal{N}\left(\frac{\mu_{\mathrm{I}, \mathrm{Y}}}{\sigma_{0}}, 1\right), \\
& \frac{R_{\mathrm{Q}}}{\sigma_{1}} \stackrel{\mid V, R_{1, \mathrm{u}}, \rho_{1, \mathrm{u}}}{\sim} \mathcal{N}\left(\frac{\mu_{\mathrm{Q}, \mathrm{Y}}}{\sigma_{0}}, 1\right) .
\end{aligned}
$$

Using the values of $\mu_{\mathrm{I}, \mathrm{Y}}$ and $\mu_{\mathrm{Q}, \mathrm{Y}}$ from (22) and (23) we get: $X_{1} \stackrel{\mid V}{\sim} \chi_{2}^{\prime 2}\left(a^{2}=\lambda_{1} \sigma_{0}{ }^{2}\right)$ and $a^{2}=\mu_{\mathrm{l}, \mathrm{Y}}{ }^{2}+\mu_{\mathrm{Q}, \mathrm{Y}^{2}}=\frac{k^{2} \rho^{2} P_{\mathrm{u}}}{2 \sigma_{0}{ }^{2} R_{1, \mathrm{u}^{2}}}$. The CCDF in terms of Marcum's $Q$ function is given as [34]:

$$
\begin{aligned}
& \bar{F}_{X_{1}}\left(x_{1} ; \mathcal{H}_{1}\right)=Q\left(a=\sqrt{\lambda_{1}}, b=\sqrt{x_{1}}\right) \\
& =\int_{\sqrt{x_{1}}}^{\infty} \exp \left\{-\frac{a^{2}+u^{2}}{2}\right\} u I_{0}(a u) \mathrm{d} u,
\end{aligned}
$$

where $I_{0}()$ represents the Modified Bessel Function of the first kind, and zero-order:

$$
\bar{F}_{R_{\mathrm{s}} \mid V, R_{1, \mathrm{u}}, \rho_{1, \mathrm{u}}}\left(r_{\mathrm{s}} ; \mathcal{H}_{0}\right)=Q\left(a=\frac{\sqrt{\lambda_{1}}}{\sigma_{0}}, b=\frac{\sqrt{r_{\mathrm{S}}}}{\sigma_{0}}\right) .
$$

Taking expectation of (54), with respect to the random variables $R_{1, \mathrm{u}}, V$, and $\rho_{1, \mathrm{u}}$ using (1) and $\rho_{1, \mathrm{u}} \sim \mathcal{U}[0,1]$, we obtain the $P_{\mathrm{FA}}=\bar{F}_{R_{\mathrm{s}}}\left(r_{\mathrm{s}} ; \mathcal{H}_{0}\right)=\mathbb{E}_{V, R_{1, \mathrm{u}}, \rho_{1, \mathrm{u}}}\left[\bar{F}_{R_{\mathrm{s}} \mid V, R_{1, \mathrm{u}}, \rho_{1, \mathrm{u}}}\left(r_{\mathrm{s}} ; \mathcal{H}_{0}\right)\right]$, which yields the expression in (34). 


\section{E. Cumulative CDFs of RSS with Alternative Distribution}

Following completely analogous steps as in Appendix D and using the distributions of $Y$ and $Z$ in Theorem 1 and Theorem 2, we derive the CCDFs of the RSS in four different cases as follows.

Case $1\left(\mathrm{~s}_{\mathrm{T}}=\mathrm{NLoS}, \mathrm{s}_{\mathrm{A}}=\mathrm{NLoS}\right)$ :

$$
\bar{F}_{R_{\mathrm{s}} \mid R_{\mathrm{d}}, V}\left(r_{\mathrm{s}} ; \mathcal{H}_{1}\right)=\exp \left(-\frac{r_{\mathrm{s}}}{2 \sigma_{1}^{2}}\right) \text {. }
$$

Case $2\left(\mathbf{s}_{T}=\right.$ NLoS, $\left.\mathrm{s}_{\mathrm{A}}=\operatorname{LoS}\right)$ :

$$
\bar{F}_{R_{\mathrm{s}} \mid R_{\mathrm{d}}, \rho_{\mathrm{d}}, V}\left(r_{\mathrm{s}} ; \mathcal{H}_{1}\right)=Q\left(a=\frac{\sqrt{\lambda_{2}}}{\sigma_{1}}, b=\frac{\sqrt{r_{\mathrm{s}}}}{\sigma_{1}}\right)
$$

Case $3\left(\mathrm{~s}_{\mathrm{T}}=\mathrm{LoS}, \mathrm{s}_{\mathrm{A}}=\mathrm{NLOS}\right)$ :

$$
\bar{F}_{R_{\mathrm{s}} \mid R_{\mathrm{d}}, \rho_{1, \mathrm{u}}, V}\left(r_{\mathrm{s}} ; \mathcal{H}_{1}\right)=Q\left(a=\frac{\sqrt{\lambda_{1}}}{\sigma_{1}}, b=\frac{\sqrt{r_{\mathrm{s}}}}{\sigma_{1}}\right) .
$$

Case $4\left(\mathrm{~s}_{\mathrm{T}}=\right.$ LoS, $\mathrm{s}_{\mathrm{A}}=$ LoS $)$ :

$$
\bar{F}_{R_{\mathrm{s}} \mid R_{\mathrm{d}}, \rho_{\mathrm{d}}, \rho_{1, \mathrm{u}}, V}\left(r_{\mathrm{s}} ; \mathcal{H}_{1}\right)=Q\left(a=\frac{\sqrt{\lambda_{3}}}{\sigma_{1}}, b=\frac{\sqrt{r_{\mathrm{s}}}}{\sigma_{1}}\right),
$$

where

$$
\lambda_{3}=\lambda_{1}+\lambda_{2}+2 \sqrt{\lambda_{1} \lambda_{2}} \cos \left(\theta_{\mathrm{d}}-\theta_{1, \mathrm{u}}\right) .
$$

Since $\theta_{\mathrm{d}} \sim \mathcal{U}[0,2 \pi)$, we have

$$
\left(\theta_{\mathrm{d}}-\theta_{1, \mathrm{u}}\right) \quad(\bmod 2 \pi) \sim U[0,2 \pi) .
$$

Thus, we have:

$$
\cos \left(\theta_{\mathrm{d}}-\theta_{1, \mathrm{u}}\right) \sim \mathcal{U}[-1,1]
$$

and

$$
\lambda_{3} \sim \mathcal{U}\left[\left(\sqrt{\lambda_{1}}-\sqrt{\lambda_{2}}\right)^{2},\left(\sqrt{\lambda_{1}}+\sqrt{\lambda_{2}}\right)^{2}\right] .
$$

Without loss of generality, we assume that $\lambda_{3}=\left(\sqrt{\lambda_{1}}+\right.$ $\left.\sqrt{\lambda_{2}}\right)^{2}$. Taking expectation of $\bar{F}_{R_{\mathrm{s}} \mid R_{\mathrm{d}}, \rho_{\mathrm{d}}, V}\left(r_{\mathrm{s}} ; \mathcal{H}_{1}\right)$, with respect to the random variables $R_{\mathrm{d}}, V$, and $\rho_{\mathrm{d}}$ using $\rho_{\mathrm{d}} \sim \mathcal{U}[0,1]$, we obtain the conditional probability of detection at a sensor that is located at a distance of $r_{\mathrm{d}}$ from the target drone,

$$
P_{\mathrm{D}}\left(r_{\mathrm{d}}\right)=\bar{F}_{R_{\mathrm{s}}}\left(r_{\mathrm{s}} \mid R_{\mathrm{d}} ; \mathcal{H}_{1}\right)=\mathbb{E}_{V, \rho_{\mathrm{d}}}\left[\bar{F}_{R_{\mathrm{s}} \mid V, R_{\mathrm{d}}, \rho_{\mathrm{d}}}\left(r_{\mathrm{s}} ; \mathcal{H}_{1}\right)\right],
$$

which yields the expression in (35).

\section{F. Detection Coverage Probability}

For a target drone at $\boldsymbol{x}_{\mathbf{d}}=\left(x_{\mathrm{d}}, y_{\mathrm{d}}, h_{\mathrm{d}}\right)$, its ground projection location is $\boldsymbol{x}_{\mathbf{d}}=\left(x_{\mathrm{d}}, y_{\mathrm{d}}\right)$. Let $\mathrm{D}$ be the event that the $n$-nearest sensor to the target drone is located at the origin $(0,0) \in \mathbb{R}^{2}$. Thus we have:

$$
\begin{aligned}
\mathbb{P}(D) & =\mathbb{P}\left(\left\|\boldsymbol{x}_{\mathbf{d}}\right\|-\frac{d r}{2} \leq R_{\mathrm{n}} \leq\left\|\boldsymbol{x}_{\mathbf{d}}\right\|+\frac{d r}{2}\right) \\
& =f_{R_{\mathrm{n}}}\left(\left\|\boldsymbol{x}_{\mathbf{d}}\right\|\right) d r .
\end{aligned}
$$

Let $\mathrm{C}$ be the event that the target drone is detected by the $n$-nearest sensor. Thus we have

$$
\mathbb{P}(C)=\mathbb{P}\left(R_{\mathrm{s}}\left(R_{\mathrm{n}}\right) \geq \gamma\right) .
$$

Let $\mathrm{E}$ be the set of all 2D locations in a ring of width $d r$, centered around the origin $(0,0)$, with inner and outer radii of $\left\|\boldsymbol{x}_{\mathbf{d}}\right\|-\frac{d r}{2}$ and $\left\|\boldsymbol{x}_{\mathbf{d}}\right\|+\frac{d r}{2}$, such that, $E=\left\{\boldsymbol{x} \in \mathbb{R}^{2}:\left\|\boldsymbol{x}_{\mathbf{d}}\right\|-\right.$ $\left.\frac{d r}{2} \leq\|\boldsymbol{x}\| \leq\left\|\boldsymbol{x}_{\mathbf{d}}\right\|+\frac{d r}{2}\right\}$. Thus using (55) and (56) we get the probability that $\forall \boldsymbol{x} \in E$, is detected by the n-nearest sensor as below

$$
\begin{aligned}
& \mathbb{P}(C \cap D)=\mathbb{P}(C \mid D) \mathbb{P}(D) \\
& =\mathbb{P}\left(R_{\mathbf{s}}\left(\left\|\boldsymbol{x}_{\mathbf{d}}\right\|\right) \geq \gamma\right) f_{R_{\mathrm{n}}}\left(\left\|\boldsymbol{x}_{\mathbf{d}}\right\|\right) d r \\
& =P_{\mathrm{D}}\left(\left\|\boldsymbol{x}_{\mathbf{d}}\right\|\right) f_{R_{\mathrm{n}}}\left(\left\|\boldsymbol{x}_{\mathbf{d}}\right\|\right) d r \\
& =\int_{\left\|\boldsymbol{x}_{\mathbf{d}}\right\|-\frac{d r}{2}}^{\left\|\boldsymbol{x}_{\mathbf{d}}\right\|+\frac{d r}{2}} P_{\mathrm{D}}\left(r_{\mathrm{n}}\right) f_{R_{\mathrm{n}}}\left(r_{\mathrm{n}}\right) d r_{\mathrm{n}} .
\end{aligned}
$$

Similarly, the coverage probability in the entire ROI is characterized as the probability that $\forall \boldsymbol{x} \in \mathbb{R}^{2}$ is detected by the n-nearest sensor, is given as below

$$
\int_{0}^{\infty} P_{\mathrm{D}}\left(r_{\mathrm{n}}\right) f_{R_{\mathrm{n}}}\left(r_{\mathrm{n}}\right) d r_{\mathrm{n}} .
$$

Substituting (2), in (57), yields the expression in (36).

\section{REFERENCES}

[1] M. M. Azari, H. Sallouha, A. Chiumento, S. Rajendran, E. Vinogradov, and S. Pollin, "Key technologies and system trade-offs for detection and localization of amateur drones," IEEE Commun. Mag., vol. 56, no. 1, pp. 51-57, 2018.

[2] M. Ezuma, F. Erden, C. K. Anjinappa, O. Ozdemir, and I. Guvenc, "Detection and classification of UAVs using RF fingerprints in the presence of Wi-Fi and Bluetooth interference," IEEE Open J. Commun. Soc., vol. 1, pp. 60-76, 2019.

[3] K. Chang, "RF sensor for interference detection-an RF signal-based drone detection system," KeySight Technologies, 2016.

[4] M. Ezuma, F. Erden, C. K. Anjinappa, O. Ozdemir, and I. Guvenc, "Micro-UAV detection and classification from RF fingerprints using machine learning techniques," IEEE Aero. Conf., pp. 1-13, 2019.

[5] R. Elhabyan, W. Shi, and M. St-Hilaire, "Coverage protocols for wireless sensor networks: Review and future directions," Journal of Communications and Networks, vol. 21, no. 1, pp. 45-60, 2019.

[6] D. Mishra and E. Natalizio, "A survey on cellular-connected uavs: Design challenges, enabling $5 \mathrm{~g} / \mathrm{b} 5 \mathrm{~g}$ innovations, and experimental advancements," Computer Networks, vol. 182, p. 107451, 2020. [Online]. Available: https://www.sciencedirect.com/science/article/pii/ S1389128620311324

[7] X. Lin, V. Yajnanarayana, S. D. Muruganathan, S. Gao, H. Asplund, H. Maattanen, M. Bergstrom, S. Euler, and Y. . E. Wang, "The sky is not the limit: Lte for unmanned aerial vehicles," IEEE Communications Magazine, vol. 56, no. 4, pp. 204-210, 2018.

[8] V. Marojevic, I. Guvenc, R. Dutta, M. L. Sichitiu, and B. A. Floyd, "Advanced wireless for unmanned aerial systems: $5 \mathrm{~g}$ standardization, research challenges, and aerpaw architecture," IEEE Vehicular Technology Magazine, vol. 15, no. 2, pp. 22-30, 2020.

[9] G. Yang, X. Lin, Y. Li, H. Cui, M. Xu, D. Wu, H. Rydén, and S. B. Redhwan, "A telecom perspective on the internet of drones: From LTEadvanced to $5 \mathrm{G}, " 2018$.

[10] I. Bor-Yaliniz, M. Salem, G. Senerath, and H. Yanikomeroglu, "Is 5g ready for drones: A look into contemporary and prospective wireless networks from a standardization perspective," IEEE Wireless Communications, vol. 26, no. 1, pp. 18-27, 2019.

[11] P. Nguyen, M. Ravindranatha, A. Nguyen, R. Han, and T. Vu, "Investigating cost-effective RF-based detection of drones," in Proceedings of the 2nd Workshop on Micro Aerial Vehicle Networks, Systems, and Applications for Civilian Use, ser. DroNet '16. New York, NY, USA: Association for Computing Machinery, 2016, p. 17-22. [Online]. Available: https://doi.org/10.1145/2935620.2935632

[12] M. U. Sheikh, F. Ghavimi, K. Ruttik, and R. Jantti, "Drone detection and classification using cellular network: A machine learning approach," in Proc. IEEE 90th Veh. Technol. Conf., Honolulu, Hawaii, Sept. 2019, pp. $1-6$. 
[13] H. Rydén, S. B. Redhwan, and X. Lin, "Rogue drone detection: A machine learning approach," in Proc. IEEE Wirel. Comm. and Net. Conf. (WCNC), Marrakech, Apr. 2019.

[14] J. Zhao, X. Fu, Z. Yang, and F. Xu, "Radar-assisted UAV detection and identification based on 5G in the internet of things," Wirel. Comm. and Mob. Comp., p. 2850263, Jul. 2019.

[15] D. Solomitckii, M. Gapeyenko, V. Semkin, S. Andreev, and Y. Koucheryavy, "Technologies for efficient amateur drone detection in 5G millimeter-wave cellular infrastructure," IEEE Commun. Mag., vol. 56 , no. 1 , pp. 43-50, 2018.

[16] V. V. Chetlur and H. S. Dhillon, "Downlink coverage analysis for a finite 3-D wireless network of unmanned aerial vehicles," IEEE Transactions on Communications, vol. 65, no. 10, pp. 4543-4558, 2017.

[17] V. V. Chetlur Ravi and H. S. Dhillon, "Downlink coverage probability in a finite network of unmanned aerial vehicle (UAV) base stations," in 2016 IEEE 17th International Workshop on Signal Processing Advances in Wireless Communications (SPAWC), 2016, pp. 1-5.

[18] M. Banagar and H. S. Dhillon, "3GPP-inspired stochastic geometrybased mobility model for a drone cellular network," in 2019 IEEE Global Communications Conference (GLOBECOM), 2019, pp. 1-6.

[19] H. Sun, X. Wang, Y. Zhang, and T. Q. S. Quek, "Performance analysis and cell association design for drone-assisted heterogeneous networks," IEEE Transactions on Vehicular Technology, vol. 69, no. 11, pp. 13741$13755,2020$.

[20] R. Amer, W. Saad, B. Galkin, and N. Marchetti, "Performance analysis of mobile cellular-connected drones under practical antenna configurations," 2020

[21] R. Amer, W. Saad, and N. Marchetti, "Toward a connected sky: Performance of beamforming with down-tilted antennas for ground and UAV user co-existence," IEEE Communications Letters, vol. 23, no. 10, pp. 1840-1844, 2019.

[22] P. Sinha, Y. Yapıcı, I. Güvenç, E. Turgut, and M. C. Gürsoy, "RSS-based detection of drones in the presence of RF interferers," in Proc. IEEE Consumer Commun. Netw. Conf. (CCNC), Las Vegas, California, Jan. 2020.

[23] A. Kammoun, H. Khanfir, Z. Altman, M. Debbah, and M. Kamoun, "Preliminary results on 3D channel modeling: From theory to standardization," IEEE Journal on Selected Areas in Communications, vol. 32 , no. 6, pp. 1219-1229, June 2014.

[24] T. D. Novlan, H. S. Dhillon, and J. G. Andrews, "Analytical modeling of uplink cellular networks," IEEE Trans. Wireless Commun., vol. 12, no. 6, pp. 2669-2679, June 2013

[25] M. Haenggi, Stochastic Geometry for Wireless Networks, 1st ed. New York, NY, USA: Cambridge University Press, 2012.

[26] A. Al-Hourani, S. Kandeepan, and S. Lardner, "Optimal LAP altitude for maximum coverage," IEEE Wireless Commun. Lett., vol. 3, no. 6, pp. 569-572, Dec 2014.

[27] P. C. Pinto and M. Z. Win, "Communication in a Poisson field of interferers-part I: Interference distribution and error probability," IEEE Trans. Wireless Commun., vol. 9, no. 7, pp. 2176-2186, July 2010.

[28] G. Stüber, Principles of Mobile Communication. Springer US, 2000.

[29] Y. S. Cho, J. Kim, W. Y. Yang, and C. G. Kang, MIMO-OFDM Wireless Communications with MATLAB. Wiley Publishing, 2010.

[30] 3GPP TR36873-c70, "Study on 3D channel model for LTE," 3rd Generation Partnership Project (3GPP), Tech. Rep., Jan. 2018.

[31] W. Davenport and W. Root, An introduction to the theory of random signals and noise, ser. Lincoln Laboratory publications. McGraw-Hill, 1958.

[32] M. Z. Win, P. C. Pinto, and L. A. Shepp, "A mathematical theory of network interference and its applications," IEEE Proceedings, vol. 97, no. 2, pp. 205-230, Feb 2009.

[33] G. Samorodnitsky and M. Taqqu, Stable Non-Gaussian Random Processes: Stochastic Models with Infinite Variance, ser. Stochastic Modeling Series. Taylor \& Francis, 1994.

[34] W. K. Pratt, "Partial differentials of Marcum's Q function," Proceedings of the IEEE, vol. 56, no. 7, pp. 1220-1221, July 1968.

[35] V. S. Abhayawardhana, I. J. Wassell, D. Crosby, M. P. Sellars, and M. G. Brown, "Comparison of empirical propagation path loss models for fixed wireless access systems," in Proc. IEEE Vehic. Technol. Conf. (VTC), vol. 1, May 2005, pp. 73-77 Vol. 1.

[36] H. ElSawy, A. Sultan-Salem, M.-S. Alouini, and M. Z. Win, "Modeling and analysis of cellular networks using stochastic geometry: A tutorial," IEEE Comm. Surv. Tutrls., vol. 19, no. 1, pp. 167-203, 2017.

[37] J. G. Andrews, A. K. Gupta, and H. S. Dhillon, "A primer on cellular network analysis using stochastic geometry," CoRR, vol. abs/1604.03183, 2016.
[38] R. W. H. Jr. and M. Kountouris, "Modeling heterogeneous network interference with using poisson point processes," CoRR, vol. abs/1207.2041, 2012.

[39] S. Mukherjee, Analytical Modeling of Heterogeneous Cellular Networks: Geometry, Coverage, and Capacity. Cambridge University Press, 2014. 